\title{
Filter Distortions in Ultra High-Throughput Satellites: Models, Parameters and Multicarrier Optimization
}

\author{
Tony Colin, Member, IEEE, Thomas Delamotte, Member, IEEE, and Andreas Knopp, Senior Fellow, IEEE
}

\begin{abstract}
Ultra high-throughput satellite systems are expected to play an essential role in future beyond $5 \mathrm{G}$ and $6 \mathrm{G}$ networks. These systems must remain as flexible as possible to adapt to heterogeneous traffic demands, while also delivering the highest possible rate for dedicated services. Satellites flexible payloads are increasingly employing wideband output multiplexers. In this context, it is now more important than ever to evaluate frequencydependent degradations on multicarrier signals. In particular, it is critical to characterize the distortions entailed by the output multiplexers filters. In this paper, models are presented and novel formulas are derived to determine the carrier-to-interference ratio resulting from these distortions. Derivations are oriented towards the applicability of either high-accuracy (e.g., for link budget) or low-complexity calculations (e.g., for real-time carrier allocation). The influence of key parameters such as the optimal decision instant, symbol rate and roll-off factor is thoroughly analyzed. Furthermore, formulas are evaluated in a practical scenario: the dynamic carrier allocation optimization. They are combined with efficient optimization algorithms to obtain the best performance based on user fairness. Relevant metrics such as accuracy, complexity and allocation gain are also investigated. In the end, the application of the proposed formulas and algorithms leads to a significant allocation gain that is increasing with the number of carriers. The feasibility of real-time dynamic carrier allocation to further increase the capacity of the next generation of satellite systems is emphasized.
\end{abstract}

Index Terms - filter distortions, satellite communications, highthroughput satellite, output multiplexer, carrier allocation, raised-cosine filter.

\section{INTRODUCTION}

$\mathbf{N}$ EARLY half of the world population is estimated not to have Internet access as reported by the latest statistics ${ }^{1}$ [2]. Thus, the main requirement of the beyond $5 \mathrm{G}$ and $6 \mathrm{G}^{2}$ endeavor is to guarantee an ubiquitous geographical coverage to connect the remaining unconnected population. For this purpose, an increased integration of satellites into terrestrial networks is envisioned [3]. The most relevant satellite applications include broadband internet services to fixed remote locations and to mobility applications (e.g., aircrafts, ships, trains, and cars) as well as complementary services via backhauling (e.g., delivery to the edge or cloud) [4]. Other use cases also include emergency and disaster relief [5].

This paper was presented in part at the 10th Advanced Satellite Multimedia Systems Conference and the 16th Signal Processing for Space Communications Workshop (ASMS/SPSC), Virtual Conference, October 2020 [1].

T. Colin, T. Delamotte and A. Knopp are with the Chair of Signal Processing, Bundeswehr University Munich, 85579 Neubiberg, Germany (email: papers.sp@unibw.de).

${ }^{1}$ The International Telecommunication Union (ITU) estimates about 3.55 billion people, i.e. $46.4 \%$ of the world's population, at the end of 2019 .

${ }^{2}$ Fifth- and sixth-generation mobile network.
Since the early 2000s, the development of high-throughput satellite (HTS) systems in a geostationary orbit have been driven by the ever-increasing demand of high data rates satellite services. The capacity of these multibeam satellite systems has been progressing to the point of passing the $1 \mathrm{Tbit} \mathrm{s}^{-1}$ mark by 2022 (e.g., ViaSat-3). Thus, they are now referred to as ultra high-throughput satellite (UHTS) systems. Two important evolutionary steps are foreseen for the next-generation UHTS systems. On the one hand, the system bandwidth will increase to meet the future target data rate of beyond $5 \mathrm{G}$ and $6 \mathrm{G}$ applications. It will be possible to obtain from $500 \mathrm{MHz}$ up to $2.9 \mathrm{GHz}$ of downlink bandwidth per polarization by shifting feeder links to the Q/V-band $(42.5-51.4 \mathrm{GHz})$ and exploiting the available Ka-band resources $(17.3-21.2 \mathrm{GHz})$ in the user links [6]. On the other hand, the allocation of the payload resources (e.g., bandwidth, time, power, and coverage) will become increasingly flexible. This will enable to adapt to different communication services and their evolution [7] One key technology of the future satellite communication payloads is the wideband output multiplexer (OMUX). The critical role of the wideband OMUX is mainly to prevent from emitting harmful out-of-band radiation [8], [9]. Novel wideband technologies offer several advantages. Firstly, it enables to reduce the complexity of the RF hardware and save dry mass [10]. Secondly, the spectrum utilization is also enhanced by eliminating guard bands between the transponder channels. Thus, it offers a multicarrier environment, where the different communication services can coexist, while still allowing for high-rate single-carrier operation. However, these technologies impose certain trade-offs in the link budget computations and carrier rate optimization.

One critical trade-off and new challenge comes from the linear distortions. They are caused by nonideal channel frequency response of the satellite filters, but do not give rise to spectral regrowth as opposed to nonlinear distortions. From a filter theory standpoint, increasing the bandwidth of the satellite filters does not change their linear distortions characteristic. However, the ratio between the carriers bandwidth and the channel bandwidth tends to decrease. This makes the carriers more prone to higher variations of distortions, especially in the presence of passband ripples and close to the edge of the filters. Therefore, it becomes crucial to determine how to optimally place these carriers within the channel bandwidth. Although these filters are typically based on Chebyshev and elliptic transfer functions [8], [11], [12], the theoretical characteristic cannot be exactly met due to challenging design constraints. From a practical standpoint, the design of the OMUX filters is especially difficult. At a physical level, they are required 
to handle high power: typically more than $120 \mathrm{~W}$ per channel [13]. Thus, the current technology inherently involves heavy and bulky hardware components. The insertion loss must also be minimized since it leads directly to a reduction of radiated power. At a signal level, the OMUX must comply with a minimum out-of-band attenuation and stringent inband specifications. In the end, this results in a complex and critical trade-off involving physical considerations and acceptable levels of linear distortions.

In the frame of this paper, two main applications requiring a novel characterization of the linear distortions have been identified. The first application lies in the initial phases of a satellite development following the widespread ECSS standard [14]. Indeed, these design phases are dedicated to identify the key drivers of the system design in terms of cost and performance. At this stage, it is of paramount importance to identify the critical carrier parameters especially in the context of the linear distortions. Satellite payload engineers, however, have to resort to empirical heritage rules to assess the linear distortions entailed by each satellite component [15]. It is then crucial to derive novel formulas enabling an accurate characterization of the linear distortions. It also leads to a clear identification of the impact of the carrier parameters. The second application consists in enabling dynamic carrier allocation (DCA) in future UHTS systems. Indeed, DCA brings key benefits. It allows a fully flexible connectivity between uplink and downlink beams, removes the need of a centralized gateway and can achieve higher capacity gains as demonstrated in terrestrial communications [16]. On top of that, it prevents from increasing the processing power onboard the satellite, which is a major drawback of alternative strategies such as beam-hopping (physical layer) or flexible routing protocols (higher layer). In fact, real-time DCA is of high interest for airborne services such as inflight entertainment, where new aircrafts appear and others disappear regularly. Thus, a lowcomplexity characterization of the critical frequency-dependent linear distortions is a decisive step towards enabling the DCA.

To date, a mathematical description of the linear distortions relevant to current and future satellite communications systems does not exist in the literature. Indeed, previous research in the field has mainly been restricted in terms of modulation technique, pulse shaping or filter distortion characteristic. For instance, one of the first groundbreaking works can be attributed to Jones [17], where the author derives an analytical expression for the bit error probability related to linear distortions, including the carrier placement with respect to the distorting filter. However, the analysis is limited to coherent binary and quadrature phase-shift keying modulations and rectangular pulse shaping. In the work of [18], the linear distortions are modeled as a third-order Butterworth filter and limited to binary frequency-shift keying modulation and rectangular pulse shaping. Moreover, the later work of [19] focuses on the error probability related to linear and nonlinear distortions using the square-root raised-cosine filter (SRRCF) as pulse shaping, but it is restricted to quadrature amplitude modulation. Consequently, scant attention has been paid to meet the characteristics of the satellite standards of today [20]-[22], i.e., based at the same time on a wide range of linear modulation techniques, SRRCF pulse shaping, and imperfect filter distortion characteristics.

In the light of the existing literature, this paper proposes a mathematical characterization of the linear distortions, which takes into account the practical needs of the satellite communications community. Thus, it extends the first investigations from [1]. The original contributions of the present paper are the following:

1) Three novel mathematical models of the typical linear distortions in UHTS systems are provided (based on poles and zeros, first-order and second-order of linear distortions). The novel and practical carrier-to-interference ratio (CIR) formulas corresponding to each model are derived.

2) The CIR expression related to the first-order of linear distortions, first formulated in [1], has been further improved. This not only leads to an even faster implementation, but also crucial new insights on the roll-off impact.

3) The impact of key parameters is thoroughly analyzed mathematically. This novel analysis brings valuable insight on realistic linear distortions behaviors and enables the use of important properties in practice such as the separation of the gain and group delay CIR.

4) The feasibility of real-time DCA optimization is demonstrated for practical applications. In this context, the novel expressions are coupled to optimization algorithms. The performance of the proposed methods is evaluated in terms of accuracy, complexity, and allocation gain.

The remainder of this paper is structured as follows. Section II covers the UHTS system description and model. In section III the typical linear distortions models are exposed. The key parameters and properties are analyzed in section IV. Section $\mathrm{V}$ contains numerical results in optimization scenarios related to the DCA. The conclusions are presented in section VI.

Notation: Vectors and matrices are denoted by lower case and upper case boldface letters, respectively. The transpose and Moore-Penrose pseudoinverse of a matrix $\mathbf{A}$ are denoted by $\mathbf{A}^{\mathrm{T}}$, and $\mathbf{A}^{\dagger}$, respectively. The modulus of a complex number $z$ are denoted $|z|$. The imaginary unit is denoted $j$ and satisfies the relation $j^{2}=-1$. $\mathbb{Z}^{*}$ represents the set of integers without the element 0. $f(n) \sim g(n)$ as $n \rightarrow 0$ means that the function $f(n)$ is asymptotically equivalent to the function $g(n)$ as $n$ tends towards small values. $\mathcal{F}[h(t)](f)$ denotes the Fourier transform of a function $h(t)$. The convolution of functions $h$ and $g$ is denoted and defined as $(h * g)(t) \triangleq \int_{\mathbb{R}} h(\tau) g(t-\tau) \mathrm{d} \tau$.

\section{System DESCRIPTION AND MODEL}

In this section, the UHTS communication system is thoroughly described. The typical linear distortions inherent to such a system are reported and located. A system model used to derive the carrier-to-interference-and-noise ratio (CINR) and, in particular, the CIR related to linear distortions is proposed.

\section{A. UHTS Communication Payload}

The forward link of a geostationary UHTS system is considered, since it is often the most demanding link. It is assumed that the satellite has a digital processor capable of processing wideband signals [23]. Although such digital processor increases the 


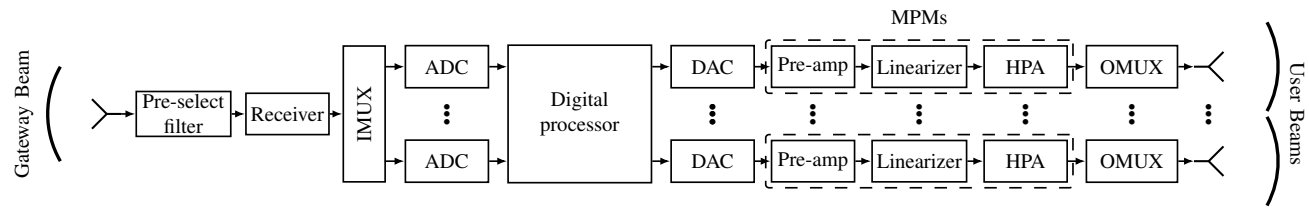

(a) UHTS communication payload.

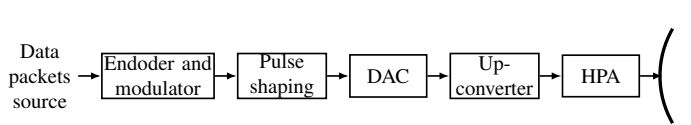

(b) Gateway transmitter.

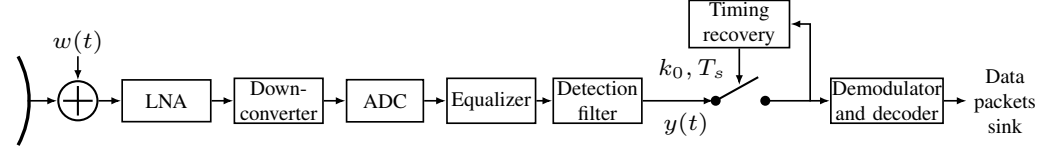

(c) User terminal receiver.

Fig. 1. Block diagrams of the future UHTS communication system. ADC indicates analog-to-digital converter; DAC, digital-to-analog converter.

power consumption, it offers significant enhancements in terms of flexibility [24], [25]. In particular, it is the key enabler of the carrier allocation optimization proposed in this paper. Thus, the UHTS communications payload depicted in Fig. 1 is considered.

Table I summarizes under which form the linear distortions typically appear in such communication payloads. Most of the components are characterized in terms of gain and group delay - equivalently amplitude and phase - by a polynomial approximation. Such approximation depends on the carrier bandwidth and placement to be considered. More generally, the gain and group delay variations are measured or estimated over the whole channel bandwidth. For instance, it is common to observe a gain slope (first-degree polynomial) in antennas, coaxes, waveguides components, whereas the group delay of these components can sometimes be described by a cubic characteristic (higher-degree polynomial) [15]. Gain and group delay ripples can arise from the mismatch between RF equipment, such as between a traveling-wave tube (TWT) and isolator within the microwave power module (MPM), as well as from multipath between components.

As opposed to the previous approximations, the complete gain and group delay characteristics of the major sources of linear distortions, most generally input multiplexer (IMUX) and OMUX, must be taken into account. A typical OMUX characteristic is depicted in Fig. 2. This characteristic is based on the DVB-S2x data points [22] and has been rescaled to a nominal bandwidth $B_{N}=500 \mathrm{MHz}$ using the recommended scaling formulas [29]. Furthermore, this characteristic is by design related to the conventional Chebyshev type II filter [30]. As a point of comparison, a fifth-order Chebyshev type II filter characteristic is provided to highlight

TABLE I

TYPICAL LINEAR DISTORTIONS CHARACTERISTICS PER PAYLOAD UNIT

\begin{tabular}{|c|c|c|c|}
\hline & Gain & Group Delay & References \\
\hline $\begin{array}{l}\text { Antennas, Coaxes, } \\
\text { Waveguides }\end{array}$ & $\begin{array}{c}\text { polynomial }^{b} \\
+ \text { ripples }\end{array}$ & $\begin{array}{c}\text { polynomial }^{b} \\
+ \text { ripples }\end{array}$ & [15] \\
\hline Receiver & polynomial $^{\mathrm{b}}$ & polynomial $^{\mathrm{b}}$ & [15] \\
\hline IMUX $^{\mathrm{a}}$ & \multicolumn{2}{|c|}{$10-4-4^{\mathrm{C}}$ Chebyshev or elliptic } & [26]-[28] \\
\hline MPM & $\begin{array}{l}\text { polynomial }^{\mathrm{b}} \\
+ \text { ripples }\end{array}$ & $\begin{array}{c}\text { polynomial }^{\mathrm{b}} \\
+ \text { ripples }\end{array}$ & [15] \\
\hline OMUX $^{\mathrm{a}}$ & \multicolumn{2}{|c|}{$6-2-0^{\mathrm{c}}$ Chebyshev or elliptic } & {$[11],[12],[28]$} \\
\hline
\end{tabular}

the asymmetry and imperfections of practical designs. Despite guiding principles in the design of IMUX and OMUX, each design is inherently unique in practice. Since analytical design techniques are more limited and computer power have been steadily increasing over the years, equipment suppliers have tended to use computer-aided optimization to achieve the final design [11], which leads to very specific locations of zeros and poles. Therefore, a precise and sufficiently flexible linear distortions characterization based on zeros and poles will be described in the next section followed by a polynomial description. In fact, linear distortions are not specific to the satellite, they also arise in the user equipment, which is often aimed to be low-cost [22]. However, these degradations are less critical than the ones of the satellite multiplexers filter. Therefore, the user equipment linear distortions will not be the focal point of the paper.

\section{B. End-to-end System Model}

At this stage, the main question consists in modeling the degradations arising between the transmitter and the receiver. Since the focus of the paper is on the linear distortions, the effects of phase noise and non-Gaussian interference (adjacent channel, cross-polar, etc.) are neglected. Furthermore, practical systems (especially in multicarrier mode) rely on high power amplifier (HPA) linearization or predistortion techniques [31], [32], which leads to a low level of nonlinearities in the system. The interaction between linear and nonlinear distortions has been described in [33]. In this work, it is highlighted that given a sufficiently low amount of nonlinearities, the linear distortions can be considered dominant. From this perspective, only the linear distortions and thermal noise are considered as signal

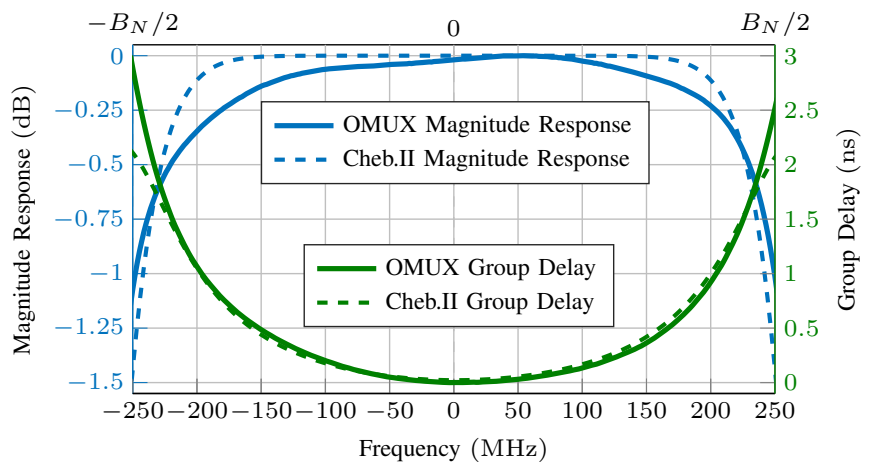

Fig. 2. Re-scaled DVB-S2x OMUX characteristic and corresponding Chebyshev type II filter model. 


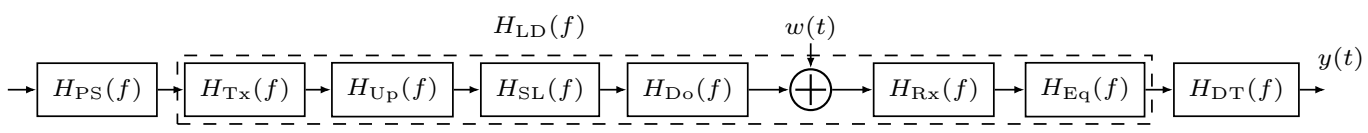

Fig. 3. Middle section of the system model. PS indicates pulse shaping; Tx, transmitter; Up, uplink channel; SL, satellite; Do, downlink channel; Rx, receiver; Eq, equalizer; DT, detection filter; LD, linear distortions.

degradation. The end-to-end system model diagram between the pulse shaping and the matched filter is illustrated in Fig. 3. This part of the system model includes the aforementioned degradations and is assumed to be linear time-invariant.

Introducing the variables, a number $N_{s}$ of modulation symbols represented by $\left\{s_{p}\right\}_{0 \leq p \leq N_{s}-1}$ is considered on the transmitter side. The impulse responses between the pulse shaping and detection filter can be factorized in a single linear distortions impulse response $h_{\mathrm{LD}}(t) \triangleq\left(h_{\mathrm{Tx}} * h_{\mathrm{Up}} * h_{\mathrm{SL}} * h_{\mathrm{Do}} *\right.$ $\left.h_{\mathrm{Rx}}\right)(t)$. The downlink noise $w(t)$ is modeled as a zero-mean white Gaussian noise of variance $\sigma^{2}$. On the receiver side, the symbols can be retrieved by sampling the signal at every period symbol $T_{s}$, i.e. at $q T_{s}$ with $q \in\left\{0,1, \ldots, N_{s}-1\right\}$. However, the optimal decision instant maximizing the system CINR is also driven by an epoch (or timing offset): $k_{0} \in \mathbb{R}$. Since the fact of introducing linear distortions leads to a distortion of the system impulse response and often to an offset of the optimal decision instant ${ }^{3}$. Therefore, a timing recovery loop is usually placed after the detection filter to track the epoch $k_{0}$ and defined relative to $T_{s}$ for the sake of generalization. This leads to the received sampled signal that can be expressed as:

$$
\begin{aligned}
& y\left(\left(k_{0}+q\right) T_{s}\right)=s_{q} \cdot h\left(k_{0} T_{s}\right)+\ldots \\
& \sum_{\substack{k=q-\left(N_{s}-1\right) \\
k \neq 0}}^{q} s_{q-k} \cdot h\left(\left(k_{0}+k\right) T_{s}\right)+w^{\prime}\left(\left(k_{0}+q\right) T_{s}\right),
\end{aligned}
$$

where $h(t) \triangleq\left(h_{\mathrm{PS}} * h_{\mathrm{LD}} * h_{\mathrm{DT}}\right)(t)$ is the system impulse response and $w^{\prime}(t) \triangleq\left(w * h_{\mathrm{Rx}} * h_{\mathrm{Eq}} * h_{\mathrm{DT}}\right)(t)$ is the filtered noise. Three terms can then be observed: the useful part of the signal, the intersymbol interference (ISI) caused by linear distortions, and the filtered noise. This constitutes the basis for the characterization of the end-to-end performance by the determination of the CINR as well as for the characterization of the linear distortions.

\section{Carrier-to-Interference-and-Noise Ratio}

Based on (1), the CINR formula can be expressed as:

$$
\mathcal{C}^{*}\left(T_{s}, \tilde{\sigma}\right)=\frac{\left|h\left(k_{0}^{*} T_{s}\right)\right|^{2}}{\sum_{k \in \mathbb{Z}^{*}}\left|h\left(\left(k_{0}^{*}+k\right) T_{s}\right)\right|^{2}+\tilde{\sigma}^{2}},
$$

where filter border effects are neglected and $\tilde{\sigma}^{2}$ represent the filtered noise power normalized by the symbols power. It will be implicitly assumed using the notation $\{\cdot\}^{*}$ that the CINR correspond to the optimal epoch $k_{0}^{*}$, which has been determined by the timing recovery loop. Indeed, the determination of $k_{0}^{*}$ depends essentially on the system impulse response $h(t)$ and

\footnotetext{
${ }^{3}$ In the practice, the epoch contains propagation delay and average group delay. It is assumed that these constants have been determined as part of the tracking and they have been subtracted from $k_{0}$, since they do not play any role in the linear distortions.
}

the constant $\tilde{\sigma}^{2}$

From this point, it is possible to decompose the CINR into:

$$
\begin{aligned}
& \left(\mathcal{C}^{*}\left(T_{s}, \tilde{\sigma}\right)\right)^{-1}=(\underbrace{\mathcal{C}_{\mathcal{I}}^{*}\left(T_{s}\right)})^{-1}+(\underbrace{\mathcal{C}_{\mathcal{N}}^{*}\left(T_{s}, \tilde{\sigma}\right)})^{-1}, \\
& \frac{\left|h\left(k_{0}^{*} T_{s}\right)\right|^{2}}{\sum_{k \in \mathbb{Z}^{*}}\left|h\left(\left(k_{0}^{*}+k\right) T_{s}\right)\right|^{2}} \frac{\left|h\left(k_{0}^{*} T_{s}\right)\right|^{2}}{\tilde{\sigma}^{2}}
\end{aligned}
$$

i.e., the CIR quantifying the degradation caused linear distortions through intersymbol interference, and the carrier-to-noise ratio (CNR) quantifying essentially the degradation caused by the noise. The SRRCF is considered in the following as pulse shaping filter and detection filter, i.e. $h_{\mathrm{PS}}(t)=h_{\mathrm{DT}}(t)=$ $h_{\mathrm{SRRCF}}(t)$. Its frequency response is defined as the square-root of the raised-cosine filter (RCF) frequency response.

\section{System Impulse Response}

The key element to determine the CIR and therefore, characterize the linear distortions, is the system impulse response $h(t)$. This impulse response can be defined as the inverse Fourier transform of the frequency responses product between the RCF and the linear distortions. The carrier center frequency is denoted $f_{\Delta}$ and defined, by convention, with respect to the center of the linear distortions characteristic. For more convenient integral calculations, the expression can be reformulated using the successive variable changes: $f^{\prime}=f-f_{\Delta}$ and $\tilde{f}=f^{\prime} \cdot T_{s}$. Thus, the system impulse response can be expressed as:

$$
h(t)=\mathcal{F}^{-1}\left[H_{\mathrm{RCF}}(\alpha, \tilde{f}) \cdot H_{\mathrm{LD}}\left(\tilde{f}+\tilde{f}_{\Delta}\right)\right](t),
$$

where the normalized carrier placement is $\tilde{f}_{\Delta}=f_{\Delta} \cdot T_{s}$ and the normalized RCF transfer function [34] is given by:

$$
\begin{aligned}
& H_{\mathrm{RCF}}(\alpha, \tilde{f})=\ldots \\
& \left\{\begin{array}{lr}
1, & |\tilde{f}| \leq \frac{(1-\alpha)}{2} \\
\frac{1}{2}\left[1+\cos \left(\frac{\pi}{\alpha}\left(|\tilde{f}|-\frac{(1-\alpha)}{2}\right)\right)\right], \frac{(1-\alpha)}{2}<|\tilde{f}| \leq \frac{(1+\alpha)}{2} \\
0, & \text { otherwise }
\end{array}\right.
\end{aligned}
$$

where $\alpha$ is the well-known roll-off taking values in the range $0 \leq \alpha \leq 1 . h(t)$ can generally be decomposed into elementary integrals each one defined on a specific frequency interval.

As a second step, knowledge on the transfer function related to linear distortions is required. By convention, payload components are characterized in terms of gain in $(\mathrm{dB})$ and group delay in (ns). Therefore, it is of interest to consider the following polar form of the transfer function:

$$
H_{\mathrm{LD}}(f)=\rho(f) \cdot e^{j \Phi(f)}
$$

where $\rho(f)$ represents the amplitude distortion and $\Phi(f)$ the phase distortion. From this expression, it is possible to define the gain (or magnitude response) as:

$$
G_{\mathrm{dB}}(f)=20 \cdot \log _{10}(\rho(f)),
$$


and the group delay as:

$$
D(f)=-\frac{1}{2 \pi} \frac{\mathrm{d} \Phi(f)}{\mathrm{d} f} .
$$

Thus, the linear distortions can be described by inverting (7) and (8) in the following manner:

$$
H_{\mathrm{LD}}(f)=e^{\xi \cdot G_{\mathrm{dB}}(f)} \cdot e^{-j 2 \pi \int D(f) \mathrm{d} f},
$$

where $\rho(f)$ is expressed as natural base exponential function by defining $\xi=\frac{\ln (10)}{20}$, which facilitates integral computations. Hence, the CINR can be precisely determined numerically in the context of satellite end-to-end performance determination as long as gain and group delay data points of components are known. The general procedure consists in the following points:

1) Summing the gain and group delay characteristics of the components considered as sources of linear distortions to obtain $G_{\mathrm{dB}}(f)$ and $D(f)$, respectively.

2) Determining the transfer function $H_{\mathrm{LD}}(f)$ from (9).

3) Performing the numerical integration of $h(t)$ in (4).

4) Computing the CINR as a function of the epoch $k_{0}$ using a partial sum in the denominator $(k \in$ $\{-L, \ldots,-1,+1, \ldots,+L\})$.

5) Optimizing the epoch $k_{0}$ so as to maximize the CINR to obtain (2).

This procedure is the most general method of determining the CINR. It relies on the aforementioned mathematical expressions as opposed to the simulation traditionally run by satellite manufacturers and equipment suppliers. However, it still gives little insight on the linear distortions behavior, e.g. with regards to the roll-off and symbol rate, and open questions: How many terms - characterized by the limit $L$ - are required to obtain a sufficiently accurate CIR? How can the epoch $k_{0}$ be properly optimized? Therefore, the next section will shed light on the numerous characteristics of linear distortions. The analysis will focus on the typical linear distortions identified in the satellite communications systems, i.e. when $H_{\mathrm{LD}}(f)$ can be described with zeros and poles or by a polynomial.

\section{TyPICAL LINEAR Distortions MODElS}

This section aims at defining the transfer function of the linear distortions and its key parameters given the selected model: pole-zero model and approximated models. The general procedure on the selection of the appropriate model of linear distortions is illustrated in Fig. 4. The basic principle can be understood as follows: the less information on the linear distortion is available, the more approximations and assumptions are required, the less accurate the model is. In this context, a trade-off is highlighted between the obtained mathematical expressions (generality, accuracy) and polynomial expressions (low-complexity, insight).

\section{A. Pole-Zero Model and Formulas}

IMUX and OMUX filters are characterized in practice by their number of poles (constraining the passband ripple), transmission zeros (constraining the stopband attenuation) and possible additional zeros (flattening the group delay). Since there are the major source of linear distortions it is reasonable to express a model given the zeros and poles. In practice, the linear distortions transfer function related to the complete

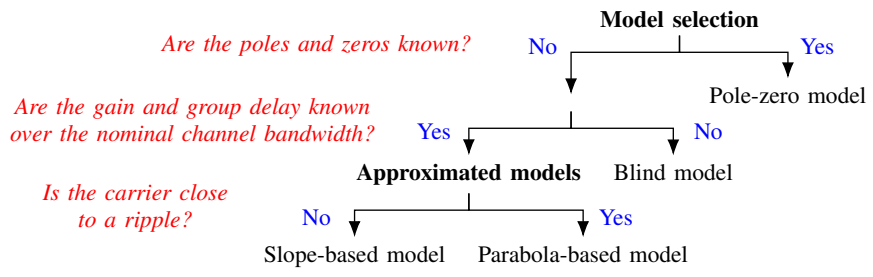

Fig. 4. Linear distortions model selection for maximum accuracy.

system will be closely related to the one of the IMUX and OMUX filters with additional perturbations.

\section{1) General derivations}

Based on (4), the system impulse response ${ }^{4}$ with regards to linear distortions can be expressed as:

$$
h\left(k_{0} T_{s}\right)=\int_{-\frac{(1+\alpha)}{2}}^{\frac{(1+\alpha)}{2}} H_{\mathrm{LD}}\left(\gamma, \tilde{f}+\tilde{f}_{\Delta}\right) H_{\mathrm{RCF}}(\alpha, \tilde{f}) e^{j 2 \pi \tilde{f} k_{0}} \mathrm{~d} \tilde{f},
$$

where

$$
H_{\mathrm{LD}}\left(\gamma, \tilde{f}+\tilde{f}_{\Delta}\right)=\frac{\prod_{i=0}^{n-1}\left(\left(\tilde{f}+\tilde{f}_{\Delta}\right)-z_{i}^{\prime}\right)}{\prod_{i=0}^{n-1}\left(\left(\tilde{f}+\tilde{f}_{\Delta}\right)-p_{i}^{\prime}\right)} .
$$

The parameters $n,\left\{z_{i}\right\}_{0<i<n-1}$ and $\left\{p_{i}\right\}_{0<i<n-1}$ are the filter order, the zeros and the poles, respectively. For the sake of compact notations, normalized poles are defined as $z_{i}^{\prime}=z_{i} / \gamma$ and $p_{i}^{\prime}=p_{i} / \gamma$. The parameter $\gamma$ is the main driver in the linear distortions behavior. It can be defined with respect to a reference frequency such as the one-sided cutoff frequency $f_{c}$. This leads to the definition $\gamma=R_{s} / f_{c}$, which is in fact a dimensionless quantity. However, to make the connection with later defined linear distortions parameters, the unit can also be interpreted as (Mbauds/MHz).

\section{2) Example of Chebyshev Type II Filter}

For the sake of illustration, the ideal Chebyshev type II filter given in Fig. 2 is considered. The zeros and poles of Chebyshev type II filters are typically of the following form:

$$
\begin{aligned}
& z_{i}=\kappa_{1} \cdot \frac{1}{\cos \left(\kappa_{2}\right)}, \\
& p_{i}=\kappa_{1} \cdot \frac{\cos \left(\kappa_{2}\right) \cosh \left(\kappa_{3}\right)+j \sin \left(\kappa_{2}\right) \sinh \left(\kappa_{3}\right)}{\cos \left(\kappa_{2}\right)^{2} \cosh \left(\kappa_{3}\right)^{2}+\sin \left(\kappa_{2}\right)^{2} \sinh \left(\kappa_{3}\right)^{2}},
\end{aligned}
$$

where $\kappa_{1}, \kappa_{2}$, and $\kappa_{3}$ are coefficients dependent on the filter order $n$ and stopband ripple $\varepsilon$. The stopband ripple can be reformulated as $\varepsilon=1 / \sqrt{A_{s}^{2}-1}$ such as to define the filter with regards to the stopband attenuation. In order to have a realistic model, $A_{s, \mathrm{~dB}}=35.0 \mathrm{~dB}$ is selected, which is above $30.0 \mathrm{~dB}$ as recommended by the DVB-S2x standard [22] and ensuring a characteristic close to the OMUX model.

\section{B. Approximated Models and Formulas}

\section{1) Least-squares problem}

From this point, it is assumed that the zeros and poles are unknown. It is also assumed that $n$ observations of the frequency response $H_{\mathrm{LD}}(f)$ gain and group delay characteristics have been obtained through measurements over the bandwidth of interest: $\left(f_{i}, G_{\mathrm{dB}, i}\right)_{1 \leq i \leq n},\left(f_{i}, D_{i}\right)_{1 \leq i \leq n}$. Gain and group

\footnotetext{
${ }^{4}$ An exponential factor has been removed, since it is not source of linear distortions.
} 


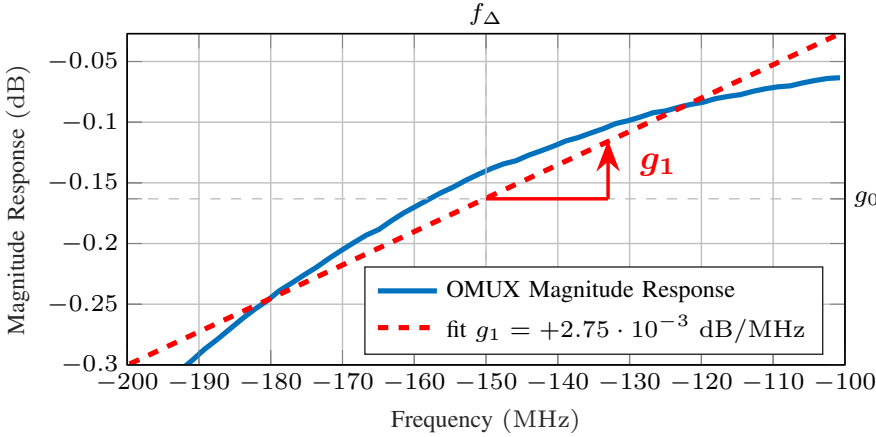

(a) Gain slope.

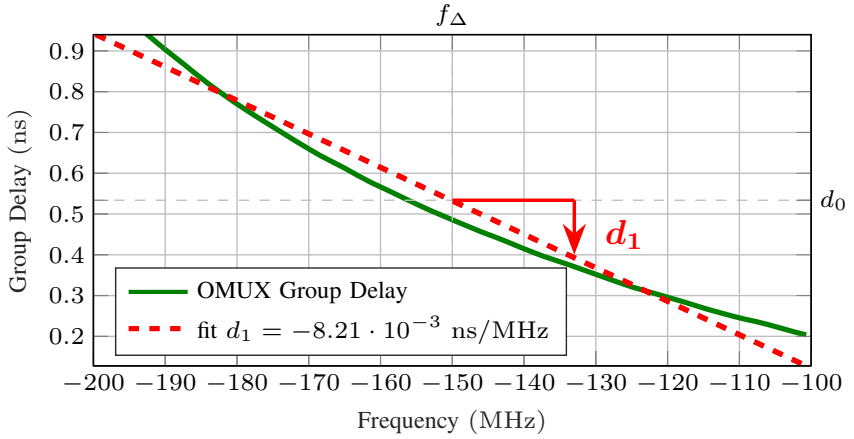

(b) Group delay slope.

Fig. 5. Example of slope fitting over a $100 \mathrm{MHz}$-carrier in the re-scaled DVB-S2x OMUX model. Selected carrier placement: $f_{\Delta}=-150 \mathrm{MHz}$.

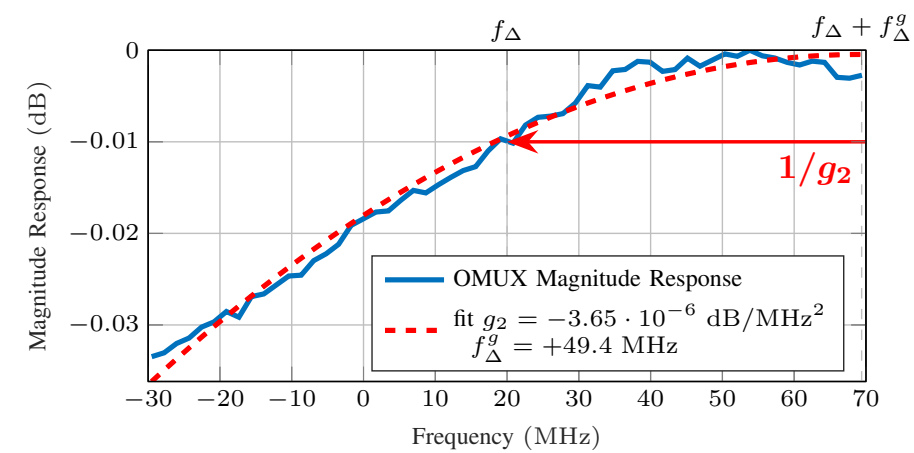

(a) Gain parabola.

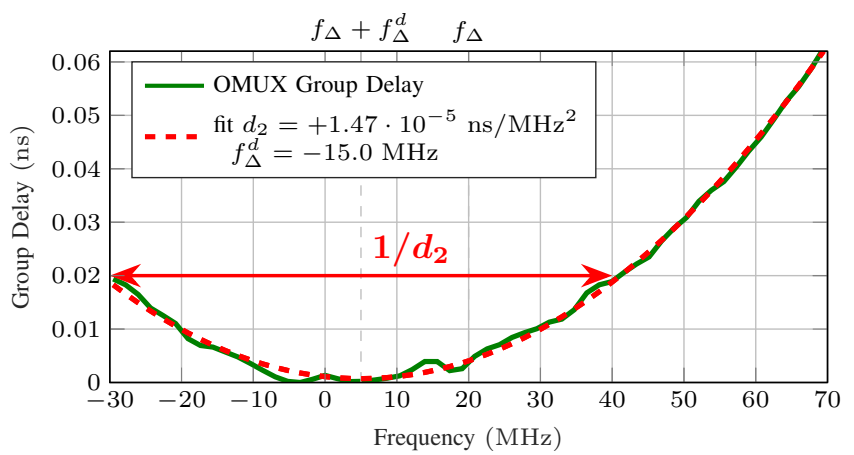

(b) Group delay parabola.

Fig. 6. Example of parabola fitting over a $100 \mathrm{MHz}$-carrier in the re-scaled DVB-S2x OMUX model. Selected carrier placement: $f_{\Delta}=+20 \mathrm{MHz}$.

delay can then be modeled as the following polynomials:

$$
\begin{aligned}
G_{\mathrm{dB}, i} & =g_{0}+g_{1} f_{i}+g_{2} f_{i}^{2}+\ldots+g_{\nu} f_{i}^{\nu}+\epsilon_{g, i}, \\
D_{i} & =d_{0}+d_{1} f_{i}+d_{2} f_{i}^{2}+\ldots+d_{\nu} f_{i}^{\nu}+\epsilon_{d, i},
\end{aligned}
$$

where $\left\{g_{p}\right\}_{0 \leq p \leq \nu},\left\{d_{p}\right\}_{0 \leq p \leq \nu}, \epsilon_{g, i}$ and $\epsilon_{d, i}$ represent the unknown polynomials coefficients of the gain and group delay, and the regression errors at the $i^{\text {th }}$-observation of the gain and group delay, respectively. $\nu$ is the degree of the gain and group delay polynomials that is later referred to as the linear distortions order $^{5}$. In matrix notations, it becomes:

$$
\begin{aligned}
\mathbf{G}_{\mathrm{dB}} & =\mathbf{F g}+\epsilon_{\mathbf{g}}, \\
\mathbf{D} & =\mathbf{F} \mathbf{d}+\epsilon_{\mathbf{d}},
\end{aligned}
$$

where $\mathbf{G}_{\mathrm{dB}}, \mathbf{D}, \mathbf{g}, \mathbf{d}$ are column vectors with elements $\left\{G_{\mathrm{dB}, i}\right\}_{1 \leq i \leq n},\left\{D_{i}\right\}_{1 \leq i \leq n},\left\{g_{p}\right\}_{0 \leq p \leq \nu}$ and $\left\{d_{p}\right\}_{0 \leq p \leq \nu}$, respectively, and $\mathbf{F}$ is a Vandermonde matrix:

$$
\mathbf{F}=\left[\begin{array}{ccccc}
1 & f_{1} & f_{1}^{2} & \ldots & f_{1}^{\nu} \\
1 & f_{2} & f_{2}^{2} & \ldots & f_{2}^{\nu} \\
\vdots & \vdots & \vdots & \ddots & \vdots \\
1 & f_{n} & f_{n}^{2} & \ldots & f_{n}^{\nu}
\end{array}\right]
$$

Finding the polynomials coefficients leads to solve quadratic minimization problems, which have the corresponding least squares (LS) solutions:

$$
\begin{aligned}
& \hat{\mathrm{g}}=\mathbf{g}^{\dagger}=\left(\mathbf{F}^{\mathrm{T}} \mathbf{F}^{-1}\right) \mathbf{F}^{\mathrm{T}} \mathbf{G}_{\mathrm{dB}} \\
& \hat{\mathbf{d}}=\mathbf{d}^{\dagger}=\left(\mathbf{F}^{\mathrm{T}} \mathbf{F}^{-1}\right) \mathbf{F}^{\mathrm{T}} \mathbf{D} .
\end{aligned}
$$

\footnotetext{
${ }^{5}$ The degree of polynomials is chosen to be the same, since it is a sufficient condition to ensure the property of independence between the gain and group delay later discussed.
}

In the end, the approximation quality of $H_{\mathrm{LD}}(f)$ is dependent on $\nu, \epsilon_{\mathbf{g}}$, and $\epsilon_{\mathbf{d}}$. In the next subsections, it is assumed that $\hat{\mathbf{g}}$ and $\hat{\mathrm{d}}$ have been determined (the notation $\hat{\imath}$ is then omitted). Core examples of LS approximation for each of the respective model are provided in Fig. 5 and 6 for the sake of illustration.

2) Slope-based model $(\nu=1)$

In the first-order linear distortions model, so-called slopebased model, the gain and group delay can be expressed as:

$$
\begin{aligned}
G_{\mathrm{dB}}\left(g_{0}, g_{1}, f\right) & =g_{0}+g_{1} f, \\
D\left(d_{0}, d_{1}, f\right) & =d_{0}+d_{1} f,
\end{aligned}
$$

which can be reduced to its linear distortions components:

$$
\begin{aligned}
G_{\mathrm{dB}}\left(g_{1}, f\right) & =g_{1} f, \\
D\left(d_{1}, f\right) & =d_{1} f,
\end{aligned}
$$

since $g_{0}$ is a scaling factor independent of the frequency, therefore not a source of linear distortions, and $d_{0}$ leads to a constant group delay, corrected by the tracking of the epoch. $g_{1}$ and $d_{1}$ are the so-called gain slope and group delay slope. Using (9), this leads to the following transfer function:

$$
H_{\mathrm{LD}}\left(g_{1}, d_{1}, f\right)=e^{\xi g_{1} f} \cdot e^{-j \pi d_{1} f^{2}},
$$

Furthermore, the final form is derived after applying the variable changes $f^{\prime}=f-f_{\Delta}$ and $\tilde{f}=f^{\prime} \cdot T_{s}$ from (4), which leads to the reformulation of the transfer function expression ${ }^{6}$ :

$$
H_{\mathrm{LD}}\left(x_{1}, y_{1}, \tilde{f}+\tilde{f}_{\Delta}\right)=e^{\xi x_{1} \tilde{f}} \cdot e^{-j \pi y_{1} \tilde{f}^{2}},
$$

where $x_{1}=g_{1} / T_{s}$ and $y_{1}=d_{1} / T_{s}^{2}$ define the two main parameters fully characterizing the behavior of the first-order

\footnotetext{
${ }^{6}$ As explained with $g_{0}$ and $d_{0}$, the terms in $\tilde{f}_{\Delta}$ are not source of linear distortions, i.e. $\tilde{f}_{\Delta}$ does not play a role in the slope-based model equations.
} 
linear distortions. Intuitively, it is expected that for a given symbol rate, increasing the gain slope worsens the CIR. Alternatively, for a given gain slope, increasing the symbol rate, i.e. the bandwidth, worsens the CIR as well. The same reasoning applies for the group delay slope. Furthermore, $x_{1}$ and $y_{1}$ are expressed in this paper in $(\mathrm{dB} / \mathrm{MHz}) \cdot(\mathrm{Mbauds})$ and $(\mu \mathrm{s} / \mathrm{MHz}) \cdot(\mathrm{Mbauds})^{2}$, respectively. These parameters are directly connected to the parameter $\gamma$ from the pole-zero model description.

3) Parabola-based model $(\nu=2)$

The gain and group delay of the second-order linear distortions, so-called parabola-based model, can be expressed as:

$$
\begin{aligned}
G_{\mathrm{dB}}\left(g_{0}, g_{1}, g_{2}, f\right) & =g_{0}+g_{1} f+g_{2} f^{2}, \\
D\left(d_{0}, d_{1}, d_{2}, f\right) & =d_{0}+d_{1} f+d_{2} f^{2},
\end{aligned}
$$

which can be reformulated more meaningfully as follows:

$$
\begin{aligned}
G_{\mathrm{dB}}\left(g_{2}, f_{\Delta}^{d}, f\right) & =g_{2}\left(f+f_{\Delta}^{g}\right)^{2}, \\
D\left(d_{2}, f_{\Delta}^{d}, f\right) & =d_{2}\left(f+f_{\Delta}^{d}\right)^{2},
\end{aligned}
$$

where $f_{\Delta}^{\{\cdot\}}$ represent the frequency offset of the parabola vertex $^{7}$ with respect to the carrier center frequency, while $1 / g_{2}$ and $1 / d_{2}$ are the latus recta of the gain and group delay parabola, respectively. The latter parameters dictate the parabola opening, i.e. the severity of the linear distortions. Similarly to the slope-based model, the linear distortions transfer function can be expressed as:

$$
\begin{aligned}
H_{\mathrm{LD}}\left(x_{2}, y_{2}, \tilde{f}_{\Delta}^{g}, \tilde{f}_{\Delta}^{d}, \tilde{f}+\tilde{f}_{\Delta}\right)= & e^{\xi x_{2}\left(\tilde{f}+\tilde{f}_{\Delta}+\tilde{f}_{\Delta}^{g}\right)^{2}} . \\
& e^{-j \frac{2 \pi}{3} y_{2}\left(\tilde{f}+\tilde{f}_{\Delta}+\tilde{f}_{\Delta}^{d}\right)^{3}},
\end{aligned}
$$

where $x_{2}=g_{2} / T_{s}^{2}$ and $y_{2}=d_{2} / T_{s}^{3}$ define the two main parameters characterizing the behavior of the second-order linear distortions. Furthermore, $x_{2}$ is expressed in this paper in $\left(\mathrm{dB} / \mathrm{MHz}^{2}\right) \cdot(\mathrm{Mbauds})^{2}$ and $y_{2}$ in $\left(\mu \mathrm{s} / \mathrm{MHz}^{2}\right) \cdot(\mathrm{Mbauds})^{3}$. Lastly, the vertexes frequency offset is also expressed with regards to the symbol period as $\tilde{f}_{\Delta}^{\{\cdot\}}=f_{\Delta}^{\{\cdot\}} \cdot T_{s}$.

\section{Parameters and Properties Analysis}

This section provides crucial new insights on typical linear distortions, with respect to the existing literature, by analyzing their behavior with regards to the key parameters: the optimal epoch $k_{0}^{*}$, the normalized noise power $\tilde{\sigma}^{2}$, the roll-off $\alpha$, the carrier placement $\tilde{f}_{\Delta}$, and the linear distortions parameters $\gamma, x_{1}, y_{1}, x_{2}, y_{2}$. Important properties are also derived. Approximated models are the center of attention, since they are the simplest form of linear distortions. A synthesis describing the typical OMUX characteristic is provided in the end of the section.

\section{A. Epoch Analysis}

\section{1) Slope-based model $(\nu=1)$}

Following the computation procedure described in section II, the CINR is determined and depicted in Fig. 7 as a function of the epoch $k_{0}$ given various sets of values $\left(x_{1}, y_{1}, \tilde{\sigma}^{2}\right)$. To comprehend the nature of the CINR and induce important properties, an analysis of its asymptotic behavior is performed.

\footnotetext{
${ }^{7}$ This equivalence can be established by setting $f_{\Delta}^{g}=g_{1} /\left(2 g_{2}\right), f_{\Delta}^{d}=$ $d_{1} /\left(2 d_{2}\right)$, and removing factors that are not source of distortions.
}

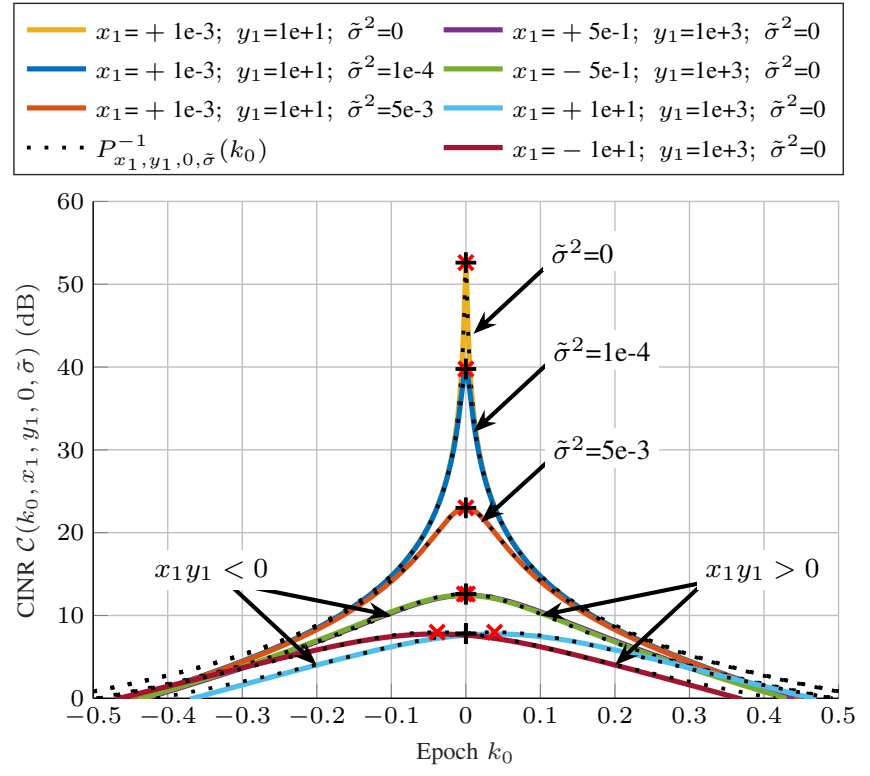

Fig. 7. Epoch influence on the slope-based model CINR. The units of $x_{1}$, and $y_{1}$ are $(\mathrm{dB} / \mathrm{MHz}) \cdot(\mathrm{Mbauds})$, and $(\mathrm{ns} / \mathrm{MHz}) \cdot(\mathrm{Mbauds})^{2}$, respectively.

Indeed, the CINR can be interpreted as the inverse of a polynomial in $k_{0}$ as follows:

$$
\mathcal{C}\left(k_{0}, x_{1}, y_{1}, \alpha, \tilde{\sigma}\right) \sim \frac{1}{P_{x_{1}, y_{1}, \alpha, \tilde{\sigma}}\left(k_{0}\right)},
$$

as $\left(k_{0}, x_{1}, y_{1}\right) \rightarrow(0,0,0), \forall \alpha \in[0 ; 1], \forall \tilde{\sigma}^{2} \geq 0$. For instance, in the case $\alpha=0$, this polynomial can be expressed as:

$$
\begin{aligned}
& P_{x_{1}, y_{1}, 0, \tilde{\sigma}}\left(k_{0}\right)=\left(\frac{1}{3}+\frac{\left(\xi x_{1}\right)^{2}}{60}+\frac{13\left(\pi y_{1}\right)^{2}}{2160}+\frac{\tilde{\sigma}^{2}}{3}\right)\left(\pi k_{0}\right)^{2}- \\
& \left(\frac{\left(\xi x_{1}\right)\left(\pi y_{1}\right)}{36}\right)\left(\pi k_{0}\right)+\left(\frac{\left(\xi x_{1}\right)^{2}}{12}+\frac{\left(\pi y_{1}\right)^{2}}{180}+\tilde{\sigma}^{2}\right) .
\end{aligned}
$$

The interested reader can refer to the appendix A for calculations details. From this expression, it is trivial to show that $P_{x_{1}, y_{1}, 0, \tilde{\sigma}}\left(k_{0}\right)$ is convex and its minimum is located at:

$$
k_{0}^{*}=\frac{\left(\xi x_{1}\right)\left(\pi y_{1}\right)}{24 \pi\left(1+\frac{\left(\xi x_{1}\right)^{2}}{20}+\frac{13\left(\pi y_{1}\right)^{2}}{720}+\tilde{\sigma}^{2}\right)},
$$

which is the optimal epoch that maximizes the CINR given the above conditions. This especially implies that the optimal epoch is not exactly zero, but a function of gain slope and group delay slope parameters $x_{1}$ and $y_{1}$. However, it can be considered that $k_{0}^{*} \sim 0$ as $\left(x_{1}, y_{1}\right) \rightarrow(0,0)$ as illustrated in Fig. 7. Moreover, it is only when the linear distortions are severe, typically for CINR $<10 \mathrm{~dB}$, that approximating $k_{0}^{*}$ to zero is no longer valid. In this case, the CINR corresponding to $x_{1} y_{1}<0$ is simply the reflection over the $y$-axis of the CINR corresponding to $x_{1} y_{1}>0$. Therefore, the maximum value is conserved and the study of the CINR can be reduced to $x_{1} \geq 0$ and $y_{1} \geq 0$. Paradoxically, the smaller $x_{1}, y_{1}$, and $\tilde{\sigma}^{2}$, the more sensitive to the epoch the CINR. Thus, the tracking of the optimal epoch is a critical matter, since even a small additive interference along with an imperfect estimation of the epoch can already significantly impact the CINR. This phenomenon was numerically observed in [35] in the presence of a non-constant group delay in the satellite communication chain. 


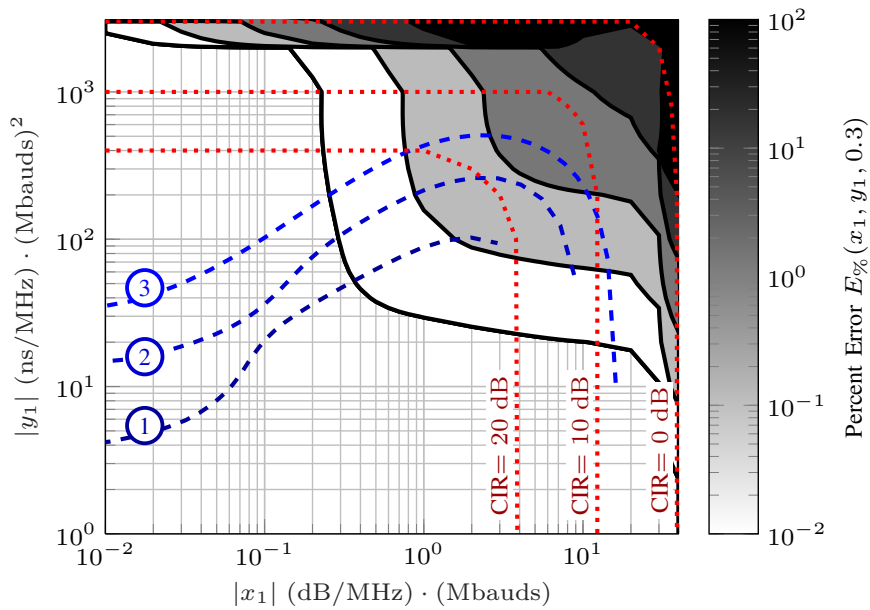

Fig. 8. Slope-based model CIR percent error $(\alpha=0.3)$. The blue dashed lines 1,2 , and 3 refer to the ranges of interest $\left(\left|x_{1}\right|,\left|y_{1}\right|\right)$ corresponding to the symbol rates: $R_{s}=0.1 B_{N}, R_{s}=0.2 B_{N}$, and $R_{s}=0.4 B_{N}$, respectively.

\section{2) Parabola-based model $(\nu=2)$}

The same study can be conducted for the parabola-based model. In this context, the case $\tilde{f}_{\Delta}^{g}=\tilde{f}_{\Delta}^{d}=0$ is of particular interest, since it corresponds to the case where the slope approximation performs poorly. Indeed, approximating a parabola by a slope at its vertex leads to the worst case of underfitting. The slope is approximated to zero, as in the case where no linear distortions are present. This leads to significantly high CINR values (tending towards infinity if $\tilde{\sigma}^{2}=0$ ) and important approximation errors.

Furthermore, the CINR can also be interpreted as the inverse of a convex polynomial in $k_{0}$. For instance, in the simpler case $\alpha=0$, the polynomial minimum is located at $k_{0}^{*} \sim y_{2} / 20$ as $\left(x_{2}, y_{2}\right) \rightarrow(0,0), \forall \tilde{\sigma}^{2} \geq 0$. It means that the optimal epoch of the centered group delay parabola is leading the optimal epoch of the parabola-based model. In other words, the main difference with the slope-based model lies in the fact that the CINR characteristic is shifted along the $k_{0}$-axis according to the value of $y_{2}$.

\section{B. Asymptotic Decomposition}

From this point, it is assumed that the normalized noise power has been taken into account and the focus is on the CIR derivations.

1) Slope-based model $(\nu=1)$

In view of the epoch study, the CIR describing the asymptotic behavior at the optimal epoch can be defined more broadly with the novel analytical expression:

$$
\mathcal{C}_{\mathcal{I}}^{*}\left(x_{1}, y_{1}, \alpha\right) \sim \underbrace{\frac{1}{\left(\xi x_{1}\right)^{2} \mathfrak{f}_{1}(\alpha)+\left(\pi y_{1}\right)^{2} \mathfrak{f}_{2}(\alpha)}}_{\mathcal{C}_{\mathcal{I}}^{\circ}\left(x_{1}, y_{1}, \alpha\right)},
$$

as $\left(x_{1}, y_{1}\right) \rightarrow(0,0), \forall \alpha \in[0 ; 1]$, where

$$
\begin{aligned}
& \mathfrak{f}_{1}(\alpha)=\sum_{k \in \mathbb{Z}^{*}} \begin{cases}\frac{\cos (\alpha \pi k)^{2}}{4(\pi k)^{2}\left((2 \alpha k)^{2}-1\right)^{2}}, & |k| \neq \frac{1}{2 \alpha} \\
\frac{1}{64 k^{2}}, & |k|=\frac{1}{2 \alpha}\end{cases} \\
& \mathfrak{f}_{2}(\alpha)=\sum_{k \in \mathbb{Z}^{*}} \begin{cases}\frac{\left(\frac{\alpha \sin (\alpha \pi k)}{\left.2(\pi k)(2 \alpha k)^{2}-1\right)}+\right.}{\left.\frac{\left(3(2 \alpha k)^{2}-1\right) \cos (\alpha \pi k)}{2(\pi k)^{2}\left((2 \alpha k)^{2}-1\right)^{2}}\right)^{2},} & |k| \neq \frac{1}{2 \alpha} \\
\frac{9}{256 \pi^{2} k^{4}}, & |k|=\frac{1}{2 \alpha}\end{cases}
\end{aligned}
$$

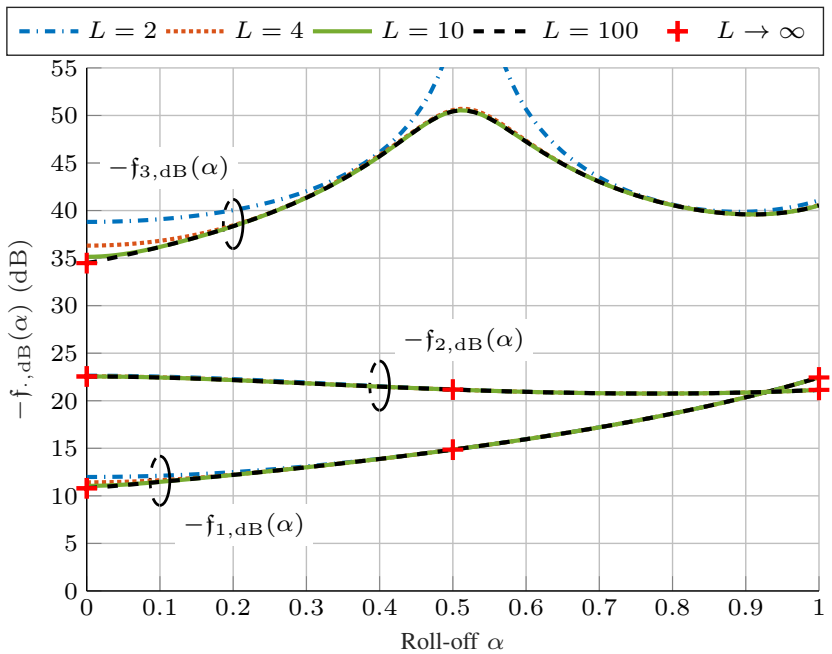

Fig. 9. Roll-off functions. $L$ indicates the partial sum limit.

In fact, each infinite sum within roll-off functions $\mathfrak{f}_{1}(\alpha)$ and $\mathfrak{f}_{2}(\alpha)$ can be explicitly determined for a given $\alpha$. For example, some particular values are $\mathfrak{f}_{1}(0)=\frac{1}{12}, \mathfrak{f}_{1}(0.5)=\frac{1}{12}-\frac{1}{2 \pi^{2}}$, $\mathfrak{f}_{2}(0)=\frac{1}{180}$, and $\mathfrak{f}_{2}(1)=\frac{17}{360}-\frac{29}{12 \pi^{2}}+\frac{20}{\pi^{4}}$. This expression enables to conclude on several properties of the CIR given the aforementioned conditions:

1) The CIR is even with respect to the gain slope and group delay slope ${ }^{8}$.

2) The impact of the roll-off $\alpha$ and symbol rate $R_{s}$ on the CIR can be considered independent of each other, when considering fixed carrier placement $\tilde{f}_{\Delta}$, gain slope $g_{1}$ and group delay slope $d_{1}$.

3) The CIR can be decomposed into a gain slope CIR, and a group delay slope CIR, where each ratio corresponds to a degradation independent of the others. In other words, no cross term $x_{1} y_{1}$ is involved.

The last property is especially important from a payload specification perspective, since it corresponds to the case where the gain slopes and the group delay slopes of the payload components can be considered independently of each other. Hence, the following composition rule can be applied:

$$
\left(\mathcal{C}_{\mathcal{I}}^{*}\left(x_{1}, y_{1}, \alpha\right)\right)^{-1} \sim(\underbrace{\mathcal{C}_{\mathcal{I}}^{*}\left(x_{1}, \alpha\right)}_{\mathcal{C}^{*}\left(x_{1}, 0, \alpha\right)})^{-1}+(\underbrace{\mathcal{C}_{\mathcal{I}}^{*}\left(y_{1}, \alpha\right)}_{\mathcal{C}^{*}\left(0, y_{1}, \alpha\right)})^{-1},
$$

$\forall \alpha \in[0 ; 1]$, as long as $\left(x_{1}, y_{1}\right) \rightarrow(0,0)$, where $\mathcal{C}_{\mathcal{I}}^{*}\left(x_{1}, \alpha\right)$ and $\mathcal{C}_{\mathcal{I}}^{*}\left(y_{1}, \alpha\right)$ are the gain slope CIR and group delay slope CIR, respectively. Furthermore, it is possible to determine the area $\left(x_{1}, y_{1}, \alpha\right)$ within which (38) holds, by studying the following CIR percent error:

$$
E_{\%}\left(x_{1}, y_{1}, \alpha\right)=\left|\frac{\mathcal{C}_{\mathcal{I}, \mathrm{dB}}^{*}\left(x_{1}, y_{1}, \alpha\right)-\mathcal{C}_{\mathcal{I}, \mathrm{dB}}^{\circ}\left(x_{1}, y_{1}, \alpha\right)}{\mathcal{C}_{\mathcal{I}, \mathrm{dB}}^{*}\left(x_{1}, y_{1}, \alpha\right)}\right| \cdot 10^{2} .
$$

An example of percent error is depicted in Fig. 8 as a grayscale, where the lighter the color, the smaller the percent error. It is considered that beyond the last contour corresponding to a percent error of $0.01 \%$, the area is free of error. Since the most common roll-off $\alpha$ belongs to the interval $[0,0.3]$, the upperbound $\alpha=0.3$ is considered as a representative value. It

$$
{ }^{8} \mathcal{C}_{\mathcal{I}}^{*}\left(-x_{1}, y_{1}, \alpha\right)=\mathcal{C}_{\mathcal{I}}^{*}\left(x_{1}, y_{1}, \alpha\right) \text { and } \mathcal{C}_{\mathcal{I}}^{*}\left(x_{1},-y_{1}, \alpha\right)=\mathcal{C}_{\mathcal{I}}^{*}\left(x_{1}, y_{1}, \alpha\right) .
$$




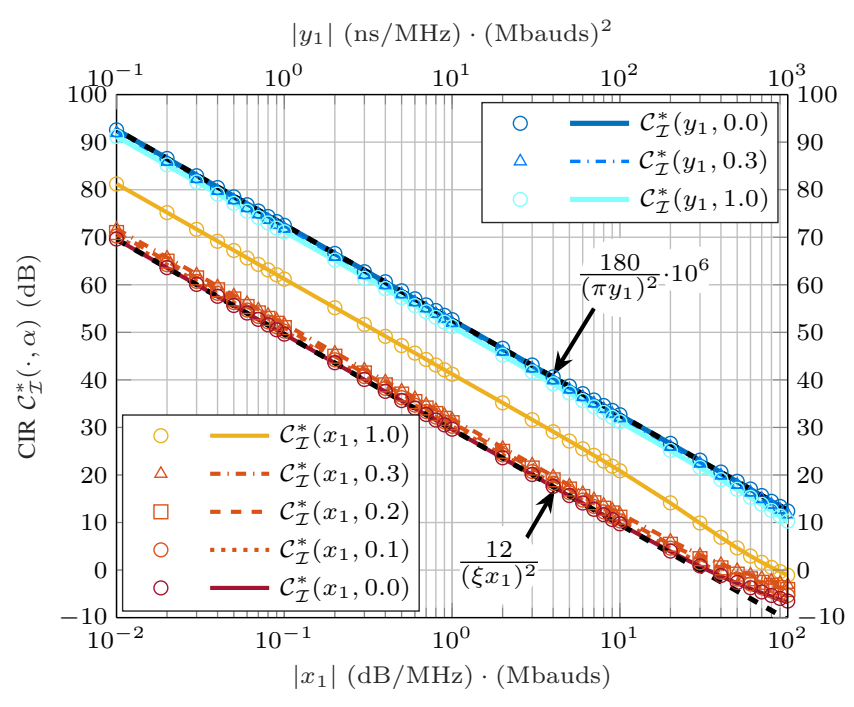

(a) Slope-based model CIR.

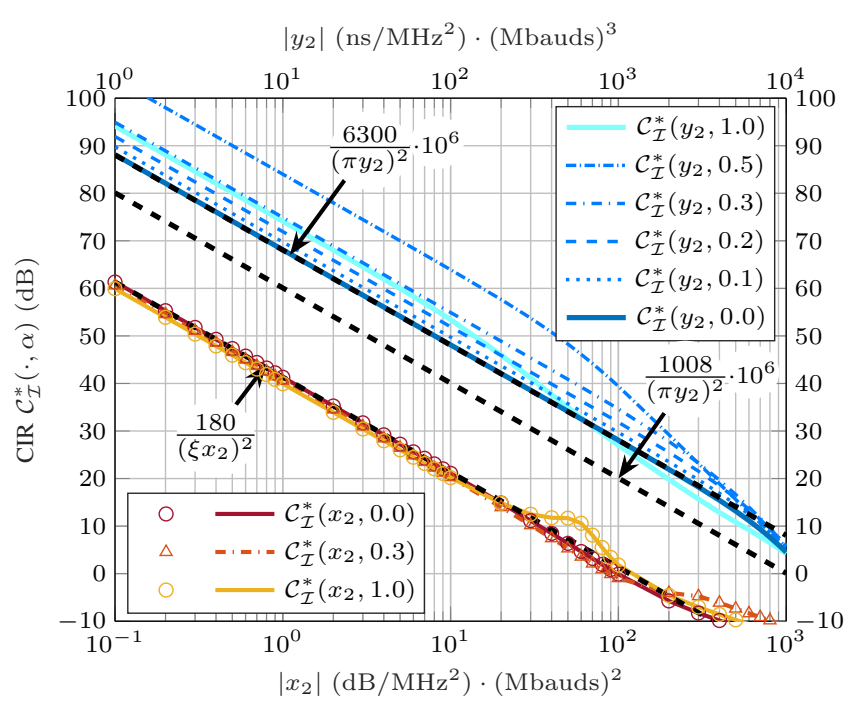

(b) Parabola-based model CIR $\left(\tilde{f}_{\Delta}^{g}=\tilde{f}_{\Delta}^{d}=0\right)$.

Fig. 10. Markers and lines represent simulations data points and theoretical curves, respectively.

is observed that for any CIR above $20 \mathrm{~dB}$ (red dotted line), the composite formula (38) leads in the worse case scenario to a percent error of about $0.5 \%$, which corresponds to a maximum approximation error of only $0.1 \mathrm{~dB}$. Moreover, this boundary is further extended for lower $\alpha$ : for $\alpha=0.2$, a maximum approximation error of $0.1 \mathrm{~dB}$ holds for any CIR above $13 \mathrm{~dB}$, whereas for $\alpha=0.1$, it corresponds to any CIR above $4 \mathrm{~dB}$. On top of that, the composite formula can also be applied when $\left|x_{1}\right|$ or $\left|y_{1}\right|$ is more dominant than the other.

To understand what can be expected in practice, the re-scaled DVB-S2x OMUX model in Fig. 2 is considered as $H_{\mathrm{LD}}(f)$. Ranges $\left(\left|x_{1}\right|,\left|y_{1}\right|\right)$ are illustrated in dashed blue lines for various ratios $R_{s} / B_{N}$ corresponding to a carrier of bandwidth $B=(1+\alpha) R_{s}$ and center frequency $f_{\Delta} \in\left[0 ;\left(B_{N}-B\right) / 2\right]$. It can be observed that for small carriers with respect to the OMUX bandwidth, i.e. small $R_{s} / B_{N}$, the composite formula can be applied with high accuracy. Typically, for $R_{s} / B_{N} \leq 0.1$, the CIR approximation error is less than $0.04 \mathrm{~dB}$. However, a carrier located at the OMUX edge with a symbol rate $R_{s} \geq 0.2 B_{N}$ has a CIR approximation error of at least $0.15 \mathrm{~dB}$. This means that the application of the composite formula must be done with care.

\section{2) Parabola-based model $(\nu=2)$}

Based on the epoch study, the asymptotic CIR at the optimal epoch can be defined, in the case $\tilde{f}_{\Delta}^{g}=\tilde{f}_{\Delta}^{d}=0$, as:

$$
\mathcal{C}_{\mathcal{I}^{*}}\left(x_{2}, y_{2}, 0,0, \alpha\right) \sim \frac{1}{\left(\xi x_{2}\right)^{2} \mathfrak{f}_{2}(\alpha)+\left(\frac{2}{3} \pi y_{2}\right)^{2} \mathfrak{f}_{3}(\alpha)},
$$

as $\left(x_{2}, y_{2}\right) \rightarrow(0,0), \forall \alpha \in[0 ; 1] . \mathfrak{f}_{2}(\alpha)$ is the same function that described the group delay slope (corresponding to a parabolic phase). In other words, this roll-off function arises from any parabolic behavior indifferently from its nature (gain or phase). On the other hand, $\mathfrak{f}_{3}(\alpha)$ arises from the parabolic group delay (corresponding to a cubic phase). This function also takes into account the correction coefficient of the optimal epoch that is dependent on $y_{2}$. Analog properties of the slopebased model can be induced (symmetry, symbol rate and roll-off independence, and composition rule) and analog conclusions can be reached regarding the CIR percent error with regards to the range $\left(\left|x_{2}\right|,\left|y_{2}\right|\right)$.

\section{Carrier Placement Analysis}

Assuming a parabola-based model, no analytical expression can be derived considering the case $\left(\tilde{f}_{\Delta}^{g}, \tilde{f}_{\Delta}^{d}\right) \in \mathbb{R}^{* 2}$ without using further approximation. It is of interest to observe the case where the carrier is near the group delay parabola vertex i.e. $\tilde{f}_{\Delta} \rightarrow-\tilde{f}_{\Delta}^{d}$. A further analytical expression can be obtained when considering $\Delta=\tilde{f}_{\Delta}^{g}-\tilde{f}_{\Delta}^{d} \rightarrow+\infty$ (assuming $\Delta>0$ ). Indeed, the transfer function (31) can be reformulated as:

$$
H_{\mathrm{LD}}\left(x_{2}, y_{2}, \tilde{f}_{\Delta}^{g}, \tilde{f}_{\Delta}^{d}, \tilde{f}+\tilde{f}_{\Delta}\right) \sim e^{\xi\left(2 x_{2} \Delta\right) \tilde{f}} \cdot e^{-j \frac{2 \pi}{3} y_{2} \tilde{f}^{3}},
$$

as long as $\left(\tilde{f}_{\Delta}, \Delta\right) \rightarrow\left(-\tilde{f}_{\Delta}^{d},+\infty\right)$. By defining $x_{1} \triangleq 2 x_{2} \Delta$, it is then trivial to see that the gain parabola can be reduced to a gain slope. Using the same reasoning and assumptions, the problem can be reduced when the carrier is near the gain parabola vertex as long as $-\Delta \rightarrow+\infty$. In practice, a distance between the vertexes of $|\Delta| \geq 0.60$ is sufficient to guarantee a percent error below $0.3 \%(\alpha \in[0 ; 0.3])$. This means that the parabola-based model is only necessary when the carrier is very close to a ripple (gain or group delay) to get a higher CIR accuracy. In other instances, the slope-based model is both accurate and of lowest complexity.

Another case of interest is $\tilde{f}_{\Delta}^{g}=\tilde{f}_{\Delta}^{d}=\tilde{f}_{\Delta}^{g, d}$, since it corresponds to the case where the both gain and group delay parabolas are aligned as in most conventional filters. By applying an analog reasoning with $\left|\tilde{f}_{\Delta}^{g, d}\right| \rightarrow+\infty$, the problem can be reduced to the first-order linear distortions. On the other hand, an analysis of $\left|\tilde{f}_{\Delta}^{g, d}\right|$ can show that the CIR is very sensitive around a vertex. For instance, a carrier shifted around the vertex by an order of $10^{-2}$ can lead to a CIR variation of the order of $\mathrm{dBs}$.

\section{Roll-off Analysis}

The roll-off functions $\mathfrak{f}_{1}(\alpha), \mathfrak{f}_{2}(\alpha)$, and $\mathfrak{f}_{3}(\alpha)$ are illustrated in Fig. 9 and expressed in (dB) unit. It is important to observe that there is no particular trend and that the optimal roll-off 


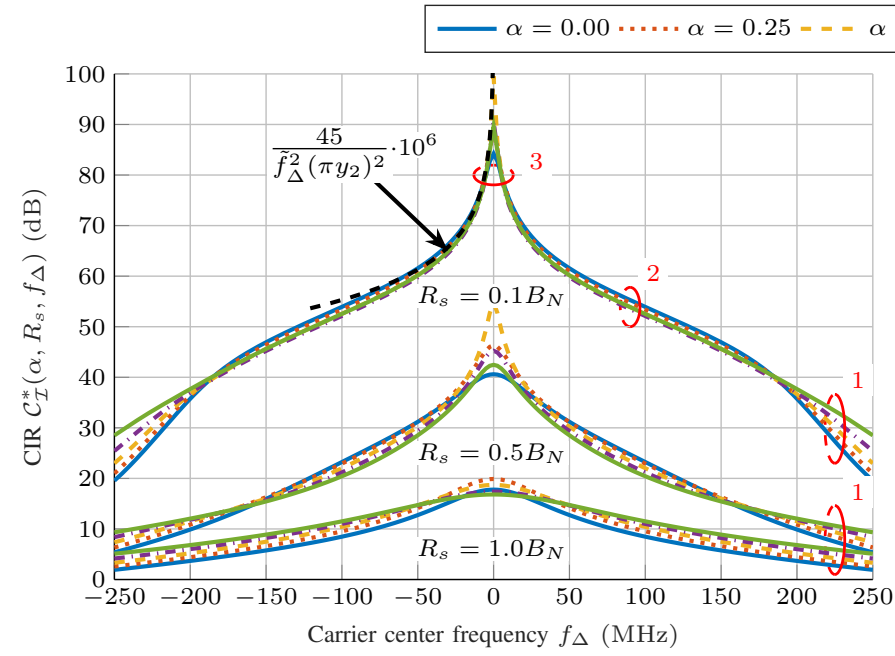

(a) Chebyshev type II model (symmetric).

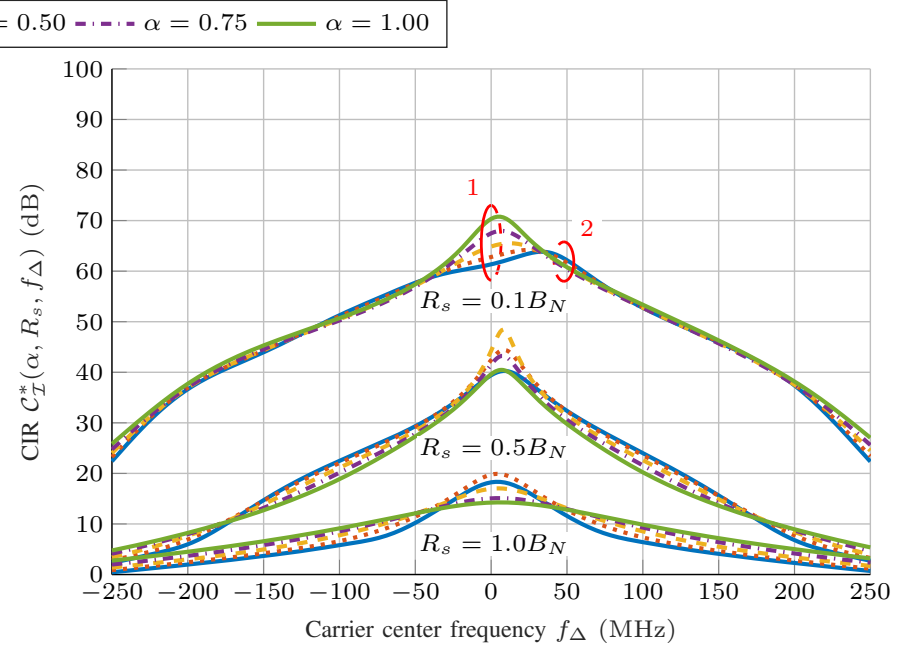

(b) Re-scaled DVB-S2x OMUX (asymmetric).

Fig. 11. CIR as a function of the carrier placement given various roll-off and symbol rates. Red circles indicates the domination of one of the following characteristic: gain slope (1), group delay slope (2), and group delay parabolic (3).

depends widely on the linear distortions order to be considered. The optimal roll-off of $\mathfrak{f}_{1}(\alpha), \mathfrak{f}_{2}(\alpha)$, and $\mathfrak{f}_{3}(\alpha)$ are $\alpha=1$, $\alpha=0$, and $\alpha \approx 0.51$, respectively. The worse roll-off value of $\mathfrak{f}_{1}(\alpha), \mathfrak{f}_{2}(\alpha)$, and $\mathfrak{f}_{3}(\alpha)$ are $\alpha=0, \alpha \approx 0.78$, and $\alpha=0$, respectively. Consequently, the optimal roll-off for a carrier (maximizing the CIR) can be widely different according to the placement of this carrier. Besides, various partial sum limits $L$ are given to bring the attention to the memory effects that are especially important for predistortion algorithms. Indeed, $\mathfrak{f}_{1}(\alpha)$ and $\mathfrak{f}_{3}(\alpha)$ display more memory for low roll-off values, but not only. $\mathfrak{f}_{3}(\alpha)$ also contains some memory around its peak. In contrast, $\mathfrak{f}_{2}(\alpha)$ convergence is fast and almost no memory is observed.

\section{E. Gain and Group Delay CIR Analysis}

\section{1) Slope-based model $(\nu=1)$}

To get the full picture on the slope-based model, it is important to study separately $\mathcal{C}_{\mathcal{I}}^{*}\left(x_{1}, \alpha\right)$ and $\mathcal{C}_{\mathcal{I}}^{*}\left(y_{1}, \alpha\right)$, especially their asymptotic behavior. A summary of their respective behavior is depicted in Fig. 10a. First of all, $\mathcal{C}_{\mathcal{I}}^{*}\left(x_{1}, \alpha\right)$ is the simplest expression among all polynomial linear distortions and the CIR can be described as the following analytical expression:

$$
\mathcal{C}_{\mathcal{I}}^{*}\left(x_{1}, \alpha\right)=\frac{1}{\left(\xi x_{1}\right)^{2} \mathfrak{f}_{1}\left(x_{1}, \alpha\right)},
$$

defined for $x_{1} \neq 0$ (the case $x_{1}=0$ is trivial), where the function $\mathfrak{f}_{1}\left(x_{1}, \alpha\right)$ is also composed of an infinite sum and has the property to be equivalent to $\mathfrak{f}_{1}(\alpha)$ as $x_{1} \rightarrow 0$. A closedform expression can be derived given any fixed value $\alpha$, for instance: $\mathfrak{f}_{1}\left(x_{1}, 0\right)=\frac{1}{2\left(\xi x_{1}\right)} \operatorname{coth}\left(\frac{\left(\xi x_{1}\right)}{2}\right)-\frac{1}{\left(\xi x_{1}\right)^{2}}$.

By contrast, $\mathcal{C}_{\mathcal{I}}^{*}\left(y_{1}, \alpha\right)$ is less obvious and can only be described as an integral-based expression due to the lack of primitive. In the case of the group delay CIR, the impact of the roll-off is less noticeable. There is only about $1.4 \mathrm{~dB}$ difference between the CIR $\alpha=0$ and $\alpha=1$ in comparison with $11.7 \mathrm{~dB}$ for the gain slope CIR.

2) Parabola-based model $(\nu=2)$

Next, a summary of the respective behavior of $\mathcal{C}_{\mathcal{I}}^{*}\left(x_{2}, \alpha\right)$ and $\mathcal{C}_{\mathcal{I}}^{*}\left(y_{2}, \alpha\right)$ is provided in Fig. $10 \mathrm{~b}$ in the case of interest
$\tilde{f}_{\Delta}^{g}=\tilde{f}_{\Delta}^{d}=0 . \mathcal{C}_{\mathcal{I}}^{*}\left(x_{2}, \alpha\right)$ is analog to $\mathcal{C}_{\mathcal{I}}^{*}\left(y_{1}, \alpha\right)$ with a rather small roll-off sensitivity and stable trend for low CIR (except around $\alpha=1)$. In contrast, regarding $\mathcal{C}_{\mathcal{I}}^{*}\left(y_{2}, \alpha\right)$, the higher the roll-off, the more unstable the CIR is. It is also highlighted (black dashed lines) that there is about $8 \mathrm{~dB}$ difference between the CIR evaluated at $k_{0}=0$ and $k_{0}^{*} \sim \frac{1}{20} \cdot y_{2}$ (at $\alpha=0$ ). This especially stresses the importance of the epoch analysis.

\section{F. Synthesis}

In Fig. $11 \mathrm{CIRs}$ are depicted as a function of the carrier placement based on the two OMUX models presented in Fig. 2. By showing the characteristic for various roll-offs, it is possible to identify linear distortions behavior from the approximated models previously described. Fig. 11a highlights that the group delay parabolic is dominant around the OMUX center for small carriers, e.g. $R_{s}=0.1 B_{N}$. Indeed, this is due to $\tilde{f}_{\Delta}^{g}=\tilde{f}_{\Delta}^{d}$ and $y_{2}$ being more dominant than $x_{2}$. The optimal roll-off is $\alpha \approx$ 0.51 , i.e. the one corresponding to $\mathfrak{f}_{3}(\alpha)$. From a normalized frequency offset corresponding to $\left|\tilde{f}_{\Delta}\right|=0.60$, i.e. $30 \mathrm{MHz}$ with respect to the OMUX center, the group delay slope can be considered dominant (with at least $99.7 \%$ accuracy). Therefore, the optimal roll-off in this range of frequencies is $\alpha=0$, i.e. the one corresponding to $\mathfrak{f}_{2}(\alpha)$. Finally, the gain slope becomes dominant further on the edge of the OMUX, where the optimal roll-off is then $\alpha=1$, i.e. the one corresponding to $\mathfrak{f}_{1}(\alpha)$. For wide carriers, e.g. $R_{s}=0.5 B_{N}$, the effect of the roll-off in the center is spread due to larger $R_{s}$. Outside the center, deviations of the roll-off effect is observed with respect to the slope-based model, since the slope approximation is less accurate with larger $R_{s} / B_{N}$. For very wide carriers, e.g. $R_{s}=1.0 B_{N}$, the optimal roll-off in the center decreases, since increasing the carrier bandwidth would result in severe degradation due to the channel bandwidth limitation.

The asymmetry of the DVB-S2x OMUX model can be well observed in Fig. 11b and especially for small carriers. Indeed, the group delay ripple and the gain ripple do not coincide, i.e. $\tilde{f}_{\Delta}^{g} \neq \tilde{f}_{\Delta}^{d}$. For small carriers, e.g. $R_{s}=0.1 B_{N}$, the normalized frequency difference between the ripples vertexes corresponds to $|\Delta| \approx 0.64$. As a result, since $|\Delta|>0.60$, a gain slope roll- 
off effect can be noticed at the group delay vertex (center-left) and a group delay slope roll-off effect can be noticed at the gain vertex (center-right). For wide carriers, e.g. $R_{s}=0.5 B_{N}$, the effect of the roll-off in the center becomes closer to the one of the Chebyshev type II model. The wider the carrier, the less difference is observed between both model.

\section{NumericAl Results IN OPTIMIZATION SCENARIOS}

This section sheds light on the feasibility of real-time DCA optimization in future UHTS systems. The DCA optimization consists in allocating multiple carriers to available parts of the spectrum given the knowledge of the frequency-dependent channel characteristics. Thus, four methods are proposed and analyzed with regards to the relevant performance metrics. The different system trade-offs are also exposed.

\section{A. Optimization Problem}

\section{1) Problem formulation}

Different optimization approaches can be considered: sumrate maximization, CIR maximization based on pre-defined user priority, or CIR maximization based on user fairness. For the sake of illustration, this paper focuses on the latter approach. Also, different approaches regarding the variables involved in the optimization are possible. The power control is considered to be independent so as to focus exclusively on the CIR optimization and related DCA gain. Thus, the DCA optimization problem can be formulated as follows:

$$
\mathcal{P}: \max _{1 \leq v_{c} \leq V_{c}}\left\{\min _{1 \leq i_{c} \leq N_{c}}\left\{\mathcal{C}_{\mathcal{I}}^{*}\left(f_{\Delta}\left(i_{c}, v_{c}\right)\right)\right\}\right\}
$$

and consists in finding the allocation $v_{c}^{*}$ that maximizes the minimum CIR of all carriers. It can also be understood as attempting to minimize the worst-case of linear distortions interference. The placement of carrier $i_{c}$ given a carriers combination $v_{c}$ is denoted $f_{\Delta}\left(i_{c}, v_{c}\right)$. The benefits of such max-min problem formulation have been especially highlighted in the context of cellular radio systems [36], [37]. It is worth noting that most resource allocation problems such as this one are NP-hard [38].

\section{2) Performance criteria}

Two major criteria come into play to compare the performance of the proposed problem-solving methods. The first criterion is the DCA execution time or complexity, which can itself be decomposed into two elementary complexities:

$$
\mathcal{O}(\mathrm{DCA}) \approx \mathcal{O}(\text { Search }) \times \mathcal{O}(\mathrm{CIR}),
$$

where $\mathcal{O}($ Search $)$ and $\mathcal{O}(\mathrm{CIR})$ represent the complexities of the search algorithm and the CIR computation, respectively. This criterion is of paramount importance, since it determines to which extent real-time DCA optimization can be applied. A naive brute-force search among all possible carrier allocations is in the order of $\mathcal{O}\left(N_{c} \cdot N_{c}\right.$ !), i.e. computationally expensive. In contrast, a smarter approach consists in computing only non-repetitive CIR values, since the placement of a given carrier is sometimes identical across different possible carrier combinations. This latter approach is used in the simulations and leads to a search complexity of $\mathcal{O}\left(N_{c} \cdot 2^{N_{c}-1}\right)$. In other words, the overall complexity comes down to the CIR complexity, which depends on the selected linear distortions model. Secondly, the optimality has to be taken into account. This criterion is a direct consequence of the accuracy of the considered CIR calculations, which also depends on the selected linear distortions model. Thus, a trade-off between CIR accuracy and complexity is presented.

\section{B. Simulations}

\section{1) Linear distortions models}

Each proposed problem-solving method is relying on a linear distortions model presented in Fig. 4. The pole-zero, hybrid (slope-based and parabola-based) and slope-based models are combined with the aforementioned optimal search algorithm. The choice of the models expose the best trade-offs between accuracy and complexity. The pole-zero model is based on the mathematical expression for best accuracy. In this context, the partial sum limit was tuned to $L=10$ such as to reduce the complexity, thus losing slightly in accuracy, but still achieving the optimal allocation gain in every scenarios. The slope-based model is based on the CIR asymptotic polynomial expression to achieve the lowest complexity possible. The roll-off functions $\mathfrak{f}_{1}(\alpha)$ and $\mathfrak{f}_{2}(\alpha)$ are precomputed for the set of expected rolloff values. The hybrid model is proposed as an enhancement of the slope-based model in terms of accuracy such that the parabola-based model is used in the vicinity of linear distortions ripples. For the sake of illustration, the blind model is defined as a heuristic, which simply places the carriers with wider bandwidths towards the center of the channel and carriers with narrower bandwidths towards the edge. This latter problemsolving method does not require search time and was based on the analysis in section IV showing that the symbol rate is the main driving parameter in the linear distortions behavior.

\section{2) Scenarios}

Thirty different randomized scenarios ${ }^{9}$ are generated for a given number of carriers $N_{c}$ to evaluate and compare the performance of the problem-solving methods. For each carrier, the symbol rate $R_{s}$ is generated following a uniform distribution. In other words, each symbol rate is chosen from the interval of remaining available bandwidth until the sum of the bandwidths is equal to $B_{N}$. This allows a good representation of different carriers characteristics present in practical systems. In order to isolate the allocation gain related to the symbol rate, the roll-off of all carriers is fixed to the standard value of $\alpha=0.1$.

\section{Numerical Results Analysis}

A reference allocation speed of the respective methods is given in Table II. The slope-based model provides the lowest complexity by a substantial margin. The pole-zero model relies on a numerical integration and epoch optimization to compute the CIR, which makes it computationally expensive. Furthermore, the hybrid model performance lies, as expected, in-between. Besides complexity, it can be noticed that the mean accuracy is strictly increasing with the number of carriers. Therefore, the statistics of $N_{c}=4$ and $N_{c}=8$ are presented in Table II. This is especially visible in the case of the hybrid

\footnotetext{
${ }^{9}$ This number of scenarios is a reasonable practical trade-off between the performance statistics and the sum of the scenarios execution time.
} 
TABLE II

ANALYSIS OF THE PROPOSED PROBLEM-SOLVING METHODS

\begin{tabular}{|c|c|c|c|c|c|}
\hline \multicolumn{3}{|c|}{ Models } & Pole-zero & Hybrid & Slope-based \\
\hline \multicolumn{3}{|c|}{ Key formulas } & (2), (11) & $(2),(31),(35)$ & $(35)$ \\
\hline \multirow{6}{*}{ 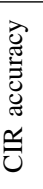 } & \multirow{3}{*}{$\begin{array}{l}\infty \\
\| \\
\end{array}$} & $\max$ & $>99.99 \%$ & $99.86 \%$ & $99.85 \%$ \\
\hline & & mean & $>99.99 \%$ & $99.73 \%$ & $99.67 \%$ \\
\hline & & $\min$ & $99.99 \%$ & $99.57 \%$ & $99.25 \%$ \\
\hline & fi & $\max$ & $>99.99 \%$ & $99.47 \%$ & $99.18 \%$ \\
\hline & \multirow{2}{*}{$z$} & mean & $99.99 \%$ & $98.61 \%$ & $98.26 \%$ \\
\hline & & $\min$ & $99.99 \%$ & $96.64 \%$ & $90.32 \%$ \\
\hline \multicolumn{3}{|c|}{ Allocation optimality } & $100.00 \%$ & $94.81 \%$ & $94.07 \%$ \\
\hline \multicolumn{3}{|c|}{ Allocation speed ${ }^{\mathrm{a}}$} & 3 & 6 & 11 \\
\hline
\end{tabular}

${ }^{a}$ Maximum carriers number where DCA can be performed under $10 \mathrm{sec}$ (execution time measured on a contemporary $3.5 \mathrm{GHz}$-processor).

and slope-based models, which are based on approximated models. Indeed, the higher the number of carriers $N_{c}$ is given a fixed OMUX bandwidth, the more often carriers have with narrower bandwidth with respect to the OMUX bandwidth. Thus, it leads to an approximation, which is more accurate statistically. Regarding the slope-based model, the smaller the carrier bandwidth is, the more the approximation $\left(x_{1}, y_{1}\right) \rightarrow(0,0)$ becomes true. As a consequence, the hybrid and slope-based models are especially well suited to a high number of carriers. It is worth noting that the accuracy of the slope-based and hybrid models are highly dependent on the linear distortions characteristics. In this context, only one gain and group delay ripple is present in the channel. By contrast, an elliptical filter containing multiple ripples would lead to a rapid decrease of the slope-based model accuracy, whereas the hybrid model accuracy would remain stable.

A comparison of the achieved minimum CIR between problem-solving methods is depicted in Fig. 12b. The allocation gain is defined as the difference between the method minimum CIR and the random allocation minimum CIR. The allocation gain depicted in Fig. 12 is significant for all considered methods. The mean allocation gain is fluctuating due to the scenarios statistics, but it is globally increasing with the number of carriers on the considered interval. For instance, the pole-zero model reaches $4.2 \mathrm{~dB}$ mean allocation gain for 4 carriers and 6.6 dB for 8 carriers. Even the proposed heuristic reaches significant mean allocation gain of $6.2 \mathrm{~dB}$ for 8 carriers. However, it is highly dependent on the linear distortions characteristics. Its performance would rapidly decrease in the presence of filter based on rippled characteristics. The measured gain optimality of the heuristic is $14.81 \%$, since the optimal DCA gain is only reached when $N_{c}$ is very small, whereas proposed methods reach at least $94.07 \%$ of the time the highest possible DCA gain.

\section{CONCLUSION}

This paper has presented a novel characterization method for the frequency-dependent filter distortions in future UHTS systems. Indeed, novel mathematical expressions of CIR have been derived depending on the available knowledge and type of filter distortions. On this basis, the crucial parameters impacting the distortions behavior have been thoroughly analyzed so as to identify and optimize the key drivers during the satellite development process. In particular, the impact of the roll-off has been shown to be dependent on the order of the filter distortions and new light has been shed on the cross-dependencies between

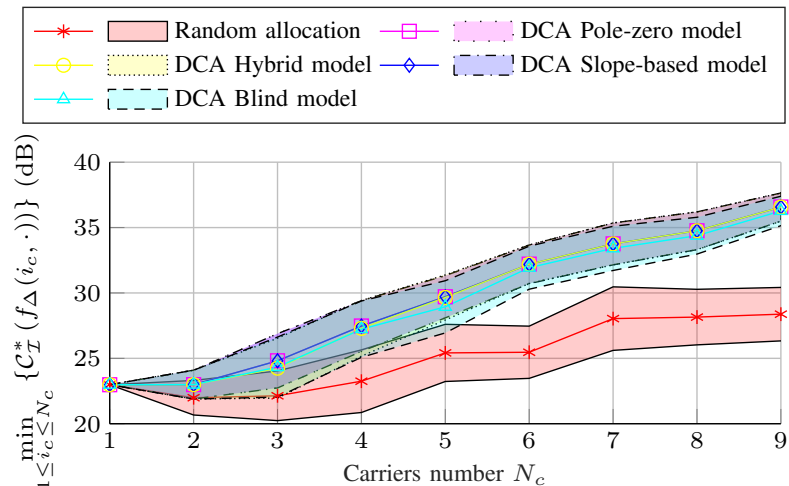

(a) Minimum CIR per scenario Vs. carrier number.

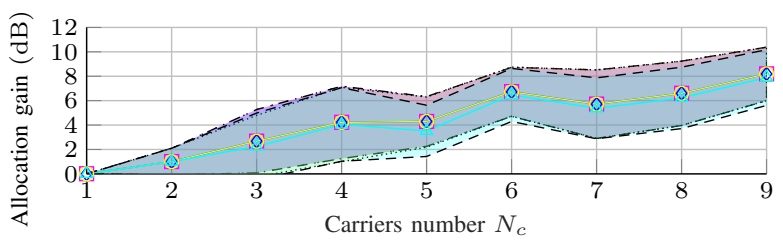

(b) Allocation gain per scenario Vs. carrier number.

Fig. 12. Performance analysis between DCA approaches. Lines and areas correspond to means and standard deviations, respectively.

gain and group delay CIRs.

Furthermore, the derived expressions are essential to enable the real-time DCA in future UHTS systems. In this context, the main trade-offs between complexity, accuracy and allocation gains have been highlighted. Thus, the proposed methods demonstrate a significant gain that is increasing with the number of carriers. In the end, this paper contributes to pave the way towards a future high-rate and seamless communication experience.

\section{APPENDIX A}

\section{Proof of the Polynomial Linear Distortions}

The first-order Maclaurin series expansion covers most of the practical cases and is developed as follows:

$$
H_{\mathrm{LD}}\left(x_{1}, y_{1}, \tilde{f}\right) \sim 1+\xi x_{1} \tilde{f}-j \pi y_{1} \tilde{f}^{2},
$$

as $\left(x_{1}, y_{1}\right) \rightarrow(0,0)$. Without loss of generality, the roll-off can be set to zero. In this case, the impulse response power at epoch $k_{0}$ can be reduced to:

$$
\left|h\left(k_{0} T_{s}\right)\right|^{2} \sim C_{0}\left(k_{0}\right)^{2}+\left(\left(\xi x_{1}\right) S_{1}\left(k_{0}\right)-\left(\pi y_{1}\right) C_{2}\left(k_{0}\right)\right)^{2},
$$

as $\left(x_{1}, y_{1}\right) \rightarrow(0,0)$, where

$$
\begin{aligned}
& C_{n}\left(k_{0}\right)=\int_{-\frac{1}{2}}^{+\frac{1}{2}} \tilde{f}^{n} \cos \left(2 \pi \tilde{f} k_{0}\right) \mathrm{d} \tilde{f}, \\
& S_{n}\left(k_{0}\right)=\int_{-\frac{1}{2}}^{+\frac{1}{2}} \tilde{f}^{n} \sin \left(2 \pi \tilde{f} k_{0}\right) \mathrm{d} \tilde{f}, n \in \mathbb{N},
\end{aligned}
$$

whose respective primitive is trivial. Then, the CINR can be determined using (2). Furthermore, a closed-form expression can be obtained by decomposing the infinite sum in the denominator into five simpler sums of the form:

$$
L_{p}\left(k_{0}\right)=\frac{1}{\pi^{p}} \sum_{k \in \mathbb{Z}^{*}} \frac{1}{\left(k+k_{0}\right)^{p}}, p \in\{2,3,4,5,6\},
$$


which can be expressed explicitly. For instance,

$$
L_{2}\left(k_{0}\right)=\frac{1}{\sin \left(\pi k_{0}\right)^{2}}-\frac{1}{\left(\pi k_{0}\right)^{2}} .
$$

The final closed-form expression can be interpreted as the inverse of the polynomial (33) when considering $k_{0} \rightarrow 0$.

\section{REFERENCES}

[1] T. Colin, T. Delamotte, R. T. Schwarz, and A. Knopp, "Linear distortions in the communication satellite payload: An analytical characterization," in 2020 10th Advanced Satellite Multimedia Systems Conference and the 16th Signal Processing for Space Communications Workshop (ASMS/SPSC), 2020, pp. 1-8.

[2] International Telecommunication Union (ITU). (2020). [Online]. Available: https://www.itu.int/en/ITU-D/Statistics/

[3] E. Yaacoub and M. Alouini, "A key 6G challenge and opportunityconnecting the base of the pyramid: A survey on rural connectivity," Proceedings of the IEEE, vol. 108, no. 4, pp. 533-582, 2020.

[4] R. De Gaudenzi, P. Angeletti, D. Petrolati, and E. Re, "Future technologies for very high throughput satellite systems," Int J Satell Commun Network, vol. 38, no. 2, pp. 141-161, 2020. [Online]. Available: https://onlinelibrary.wiley.com/doi/abs/10.1002/sat.1327

[5] F. Völk, R. T. Schwarz, M. Lorenz, and A. Knopp, "Emergency 5G communication on-the-move: Concept and field trial of a mobile satellite backhaul for public protection and disaster relief," International Journal of Satellite Communications and Networking. [Online]. Available: https://onlinelibrary.wiley.com/doi/abs/10.1002/sat.1377

[6] O. Vidal, G. Verelst, J. Lacan, E. Alberty, J. Radzik, and M. Bousquet, "Next generation high throughput satellite system," in 2012 IEEE First AESS European Conference on Satellite Telecommunications (ESTEL), 2012, pp. 1-7.

[7] H. Fenech, L. Roux, A. Hirsch, and V. Soumpholphakdy, "Satellite antennas and digital payloads for future communication satellites: The quest for efficiencies and greater flexibility," IEEE Antennas and Propagation Magazine, vol. 61, no. 5, pp. 20-28, 2019.

[8] G. Maral and M. Bousquet, "The communication payload," in Satellite Communications Systems: Systems, Techniques and Technologies, 5th ed., ser. Wiley Series in Communication and Distributed Systems. Wiley, 2009 , ch. 10 , pp. $435-525$.

[9] Unwanted emissions in the out-of-band domain, Spectrum management, International Telecommunication Union (ITU), August 2015. [Online] Available: https://www.itu.int/rec/R-REC-SM/en

[10] A. E. Atia, "Wideband communication satellite payload architecture," in 2011 IEEE MTT-S International Microwave Symposium, 2011, pp. 1-4.

[11] R. I. Cameron and M. Yu, "Design of manifold-coupled multiplexers," IEEE Microwave Magazine, vol. 8, no. 5, pp. 46-59, 2007.

[12] V. Singh, K. S. Parikh, S. Singh, and R. B. Bavaria, "DR OMUX for satellite communications: A complete step-by-step design procedure for the C-band dielectric resonator output multiplexer," IEEE Microwave Magazine, vol. 14, no. 6, pp. 104-118, 2013.

[13] M. Kunes, "Microwave multiplexers for space applications," Electronics Communication Engineering Journal, vol. 10, no. 1, pp. 29-35, 1998.

[14] Space engineering, Communications, European Cooperation for Space Standardization (ECSS), March 2021. [Online]. Available: https://ecss.nl/standard/

[15] T. M. Braun, "Payload's communications parameters," in Satellite Communications Payload and System. John Wiley \& Sons, Inc., 2012, ch. 7, pp. 181-205.

[16] I. Katzela and M. Naghshineh, "Channel assignment schemes for cellular mobile telecommunication systems: a comprehensive survey," IEEE Personal Communications, vol. 3, no. 3, pp. 10-31, 1996.

[17] J. Jones, "Filter distortion and intersymbol interference effects on PSK signals," IEEE Transactions on Communication Technology, vol. 19, no. 2, pp. 120-132, 1971 .

[18] R. Bobilin and J. Lindenlaub, "Distortion analysis of binary FSK," IEEE Transactions on Communication Technology, vol. 19, no. 4, pp. 478-486, 1971.

[19] K. Metzger and R. Valentin, "Intersymbol interference due to linear and nonlinear distortion," IEEE Transactions on Communications, vol. 44 , no. 7, pp. 809-816, 1996.

[20] Flexible Advanced Coding and Modulation Scheme For High Rate Telemetry Applications, Blue Book, Issue 1, Consulative Committee for Space Data Systems (CCSDS), March 2012. [Online]. Available: https://public.ccsds.org/
[21] Digital Video Broadcasting (DVB); DVB-SH Implementation Guidelines, European Telecommunications Standards Institute (ETSI), December 2008. [Online]. Available: https://www.etsi.org/

[22] Digital Video Broadcasting (DVB); Implementation guidelines for the second generation system for Broadcasting, Interactive Services, News Gathering and other broadband satellite applications; Part 2: S2 Extensions $(D V B-S 2 X)$, European Telecommunications Standards Institute (ETSI), November 2015. [Online]. Available: https://www.etsi.org/

[23] P. Angeletti, G. Gallinaro, L. Hili, and X. Maufroid, "Evolution of analog to digital conversion technology for wideband space applications," in 23rd AIAA International Communications Satellite Systems Conference 2005 (ICSSC).

[24] X. Maufroid, F. Coromina, B.-M. Folio, H. Göckler, H. Kopmann, and M. N. Abdulazim, "High throughput bent-pipe processor for future broadband satellite access networks," in 8th Int. Workshop on Sign. Proc. Space Commun., 2003, pp. 259-275.

[25] P. Angeletti, R. D. Gaudenzi, and M. Lisi, "From "bent pipes" to "software defined payloads": Evolution and trends of satellite communications systems," in 26th International Communications Satellite Systems Conference 2008 (ICSSC). [Online]. Available: https://arc.aiaa.org/doi/abs/10.2514/6.2008-5439

[26] F. Li, J. Yang, and X. Yin, "High quality TE 011 mode cavity filter for input multiplexer applications," in 2018 48th European Microwave Conference (EuMC), 2018, pp. 101-104.

[27] M. Latif and A. U. Salfi, "Design of 5-channel C-band input multiplexer for communication satellites," Journal of Space Technology, vol. V, no. 1, 2015.

[28] J. S. Galaz, A. P. del Pino, and P. M. Iglesias, "High order RF filters for communications satellite systems," in IWMF 2015 - 6th CNES/ESA International Workshop on Microwave Filters, 2015.

[29] Digital Video Broadcasting (DVB); Second generation framing structure, channel coding and modulation systems for Broadcasting, Interactive Services, News Gathering and other broadband satellite applications; Part 1: DVB-S2, European Telecommunications Standards Institute (ETSI), July 2014. [Online]. Available: https://www.etsi.org/

[30] R. J. Cameron, "General coupling matrix synthesis methods for chebyshev filtering functions," IEEE Transactions on Microwave Theory and Techniques, vol. 47, no. 4, pp. 433-442, 1999.

[31] B. F. Beidas, "Adaptive digital signal predistortion for nonlinear communication systems using successive methods," IEEE Transactions on Communications, vol. 64, no. 5, pp. 2166-2175, 2016.

[32] O. B. Usman and A. Knopp, "Digital predistortion in high throughput satellites: Architectures and performance," IEEE Access, vol. 9, pp. 42 291-42 304, 2021

[33] T. Colin, T. Delamotte, and A. Knopp, "Distortions characterization for dynamic carrier allocation in ultra high-throughput satellites," in 2021 29th European Signal Processing Conference (EUSIPCO), 2021, to be published. [Online]. Available: http://arxiv.org/abs/2105.11803

[34] J. G. Proakis and M. Salehi, "Digital communication through bandlimited channels," in Digital Communications, 5th ed. McGraw-Hill, 2008, ch. 9, pp. 597-688.

[35] B. M. Shankar, R. Piazza, and S. Cioni, "On-ground signal processing techniques enabling efficient amplification of multicarriers," in Cooperative and Cognitive Satellite Systems. Academic Press, 2015, ch. 4, pp. 119-153.

[36] D. J. Goodman, S. A. Grandhi, and R. Vijayan, "Distributed dynamic channel assignment schemes," in IEEE 43rd Vehicular Technology Conference, 1993, pp. 532-535.

[37] S. A. Grandhi, R. D. Yates, and D. J. Goodman, "Resource allocation for cellular radio systems," IEEE Transactions on Vehicular Technology, vol. 46, no. 3, pp. 581-587, 1997.

[38] E. L. Lawler, Recent Results in the Theory of Machine Scheduling. Berlin, Heidelberg: Springer Berlin Heidelberg, 1983, pp. 202-234. 